\title{
Lyapunov analysis for fully developed homogeneous isotropic turbulence
}

Received: 7 November 2009 / Accepted: 9 September 2010 / Published online: 30 October 2010 (C) The Author(s) 2010. This article is published with open access at Springerlink.com

\begin{abstract}
The present work studies the isotropic and homogeneous turbulence for incompressible fluids through a specific Lyapunov analysis. The analysis consists in the calculation of the velocity fluctuation through the Lyapunov theory applied to the local deformation using the Navier-Stokes equations, and in the study of the mechanism of energy cascade through the finite scale Lyapunov analysis of the relative motion between two particles. The analysis provides an explanation for the mechanism of energy cascade, leads to the closure of the von Kármán-Howarth equation, and describes the statistics of the velocity difference. Several tests and numerical results are presented.
\end{abstract}

Keywords Lyapunov analysis · Von Kármán-Howarth equation · Velocity difference statistics

\section{Introduction}

We present a novel procedure, based on a specific Lyapunov analysis, for studying the incompressible isotropic and homogeneous turbulence in an infinite domain. The analysis is mainly motivated by the fact that, in turbulence, the kinematics of the fluid deformation exhibits a chaotic behavior and huge mixing [1,2], resulting to be much more rapid than the fluid state variables. This characteristics implies that the accepted kinematical hypotheses for deriving the Navier-Stokes equations could require the consideration of very small length scales and times for describing the fluid motion [3] and therefore a very large number of degrees of freedom.

As well known, other peculiar characteristics of the turbulence are the mechanism of energy cascade, directly related to the relative motion of a pair of fluid particles [4-7] and responsible for the shape of the developed energy spectrum, and the non-gaussian statistics of the velocity difference.

This energy spectrum can be calculated through a proper closure of the von Kármán-Howarth equation (see "Appendix A") or of its Fourier Transform [6,7]. The equation, describing the evolution of the correlation function $f$ of the longitudinal velocity $u_{r}$, depends upon the term $K$, directly related to the longitudinal triplevelocity correlation $k$. Due to the inertia forces, this latter does not change the fluid kinetic energy and satisfies the detailed conservation of energy [7] which states that the exchange of energy between wave-numbers is only related to the amplitudes of these wave-numbers and of their difference [8].

Various authors (see for instance [9-11]) propose the following diffusion approximation

$$
k=2 \frac{D}{u} \frac{\partial f}{\partial r}
$$

Communicated by Spalart

N. de Divitiis $(\varangle)$

Dipartimento di Ingegneria Meccanica e Aerospaziale, University "La Sapienza", via Eudossiana 18, Rome, Italy

E-mail: dedivitiis@dma.dma.uniroma1.it 
where $r$ and $D=D(r)$ are the separation distance and the turbulent diffusivity, whereas $u^{2}=\left\langle u_{i} u_{i}\right\rangle / 3$ represents the longitudinal velocity standard deviation. This closure makes the von Kármán-Howarth equation a parabolic equation in any case (also for $v=0$ ).

To the author knowledge, Hasselmann in 1958 [9] was the first that proposed a link between $k$ and $f$, using a simple model which expresses $k$ in function of the momentum convected through the surface of a spherical volume. His model incorporates a free parameter and expresses $D(r)$ by means of a complex expression.

Another closure model in the framework of Eq. (1) was developed by Millionshtchikov [10]. There, the author assumes that $D(r)=k_{1} u r$, where $k_{1}$ is an empirical constant. Although both the models describe two possible mechanisms of energy cascade, in general, do not satisfy some physical conditions. For instance, the model of Hasselmann does not verify the continuity equation for all the initial conditions, whereas the Millionshtchikov's model gives, according to Eq. (1), a value of the skewness of $\partial u_{r} / \partial r$ in contrast with the several experiments and with the energy cascade [7].

More recently, Oberlack and Peters [11] suggested a closure model where $D$ is in terms of $f$, i.e. $D(r)=$ $k_{2} r u \sqrt{1-f}$ and $k_{2}$ is a constant parameter. The authors show that this closure reproduces the energy cascade and, for a proper choice of $k_{2}$, provides results [11] in agreement with the experimental data of the literature.

In general, Eq. (1) represents models of diffusion approximation based on the assumption that the turbulence can be viewed as diffusivity which varies with $r$. As a consequence of Eq. (1), $K$ contains a term proportional to $\partial^{2} f / \partial r^{2}$ which can occur if the velocity field includes stochastic external terms, independent from the fluid state variables $[12,13]$ and not present in the classical formulation $[6,7]$. For this reason, the models based on Eq. (1) represent phenomenological closure of Eq. (72).

Although several other works on the von Kármán-Howarth equation were written [14-17], to the author's knowledge a theoretical analysis based on basic principles which provides a physical-mathematical closure of the von Kármán-Howarth equation and the statistics of $\Delta u_{r} \equiv u_{r}(x+r)-u_{r}(x)$ has not received the due attention. Therefore, the aim of the present work is to develop a theoretical analysis based on reasonable physical conjectures which allows the closure of the von Kármán-Howarth equation and the determination of the statistics of $\Delta u_{r}$. We analyze the fully developed homogeneous isotropic turbulence in given conditions and we do not consider the intermediate stages of the turbulence.

In Sect. 2, we study the velocity fluctuation through the kinematics of the local deformation and the momentum equations. These latter are expressed with respect to the referential coordinates, which coincide with the material coordinates for a given fluid configuration [3], whereas the kinematics of the local deformation is analyzed with the Lyapunov theory. The choice of referential coordinates allows the velocity fluctuations to be analytically expressed in terms of Lyapunov exponent of the local fluid deformation.

The Sect. 3 analyzes the velocity difference between two fixed points of the space studying the motion of particles crossing the two points, in the finite scale Lyapunov basis. This basis is formally obtained through the Gram-Schmidt orthonormalization method of the finite scale Lyapunov vectors. These vectors, also known as Bred vectors, were first introduced by Toth and Kalnay [18] to study the evolution in the time of a nonlinear perturbed model subjected to an initial finite perturbation. The choice of such vectors, whose properties are related to the classical Lyapunov vectors [19], is revealed to be an useful tool for representing the relative motion of two particles crossing two given points of the space.

The present analysis postulates that the motion of such Lyapunov basis and that of the fluid with respect to the same basis, are completely statistically uncorrelated. This crucial assumption arises from the condition of fully developed turbulence. The study leads to the closure of the von Kármán-Howarth equation [6] and gives an explanation of the mechanism of kinetic energy transfer between length scales. The obtained expression of $K$ does not correspond to a diffusive approximation with model free parameters, and its mathematical structure, depending upon $f$ and $\partial f / \partial r$, satisfies the conservation law which states that the inertia forces only transfer the kinetic energy [6,7], and makes the closed von Kármán-Howarth equation a nonlinear partial differential equation of the first order in $r$ when $v=0$. The main asset of the proposed closure compared to the other models is that it has been derived from a specific Lyapunov theory, with reasonable basic assumptions about the statistics of $\Delta u_{r}$.

Furthermore, in Sect. 6, we analyze the statistics of $\Delta u_{r}$ with the Fourier analysis of the velocity fluctuations, and we obtain an analytical expression of $\Delta u_{r}$ and of its PDF. This expression incorporates an unknown function, related to the skewness, and identified through the obtained expression of $K$. This expression of $\Delta u_{r}$ also requires the knowledge of the critical Reynolds number whose estimation is made in the "Appendix B".

Finally, the comparison of the present results with the data existing in the literature indicates that the proposed Lyapunov analysis adequately describes the various properties of the fully developed turbulence. 


\section{Lyapunov analysis of the velocity fluctuations}

In this section, we analyze the velocity fluctuations through the Lyapunov analysis of the kinematic of the fluid strain, using the Navier-Stokes equations.

Starting from the momentum Navier-Stokes equations written in the frame of reference $\Re$

$$
\frac{\partial u_{k}}{\partial t}=-\frac{\partial u_{k}}{\partial x_{h}} u_{h}+\frac{1}{\rho} \frac{\partial T_{k h}}{\partial x_{h}}
$$

consider the map $\chi: \mathbf{x}_{0} \rightarrow \mathbf{x}$, which is the function that determines the current position $\mathbf{x}$ of a fluid particle located at the referential position $\mathbf{x}_{0}$ at $t=t_{0}$ [3]. Equation (2) can be written in terms of the referential position $\mathbf{x}_{0}[3]$

$$
\frac{\partial u_{k}}{\partial t}=\left(-\frac{\partial u_{k}}{\partial x_{0 p}} u_{h}+\frac{1}{\rho} \frac{\partial T_{k h}}{\partial x_{0 p}}\right) \frac{\partial x_{0 p}}{\partial x_{h}}
$$

where $T_{k h}$ represents the stress tensor. The Lyapunov analysis of the fluid strain provides the expression of this deformation in terms of maximal Lyapunov exponent $\Lambda=\max \left(\Lambda_{1}, \Lambda_{2}, \Lambda_{3}\right)$

$$
\frac{\partial \mathbf{x}}{\partial \mathbf{x}_{0}} \approx \mathrm{e}^{\Lambda\left(t-t_{0}\right)}
$$

where $\Lambda_{i},(i=1,2,3)$ are the Lyapunov exponents. Due to the incompressibility, $\Lambda_{1}+\Lambda_{2}+\Lambda_{3}=0$, thus, $\Lambda>0$.

The momentum equations written using the referential coordinates allow to factorize the velocity fluctuation and to express it in Lyapunov exponential form of the local fluid deformation. If we assume that this deformation is much more rapid than $\partial T_{k h} / \partial x_{0 p}$ and $\partial u_{k} / \partial x_{0 p} u_{h}$, the velocity fluctuation can be obtained from Eq. (3), where $\partial T_{k h} / \partial x_{0 p}$ and $\partial u_{k} / \partial x_{0 p} u_{h}$ are supposed to be constant with respect to the time

$$
u_{k} \approx \frac{1}{\Lambda}\left(-\frac{\partial u_{k}}{\partial x_{0 p}} u_{h}+\frac{1}{\rho} \frac{\partial T_{k h}}{\partial x_{0 p}}\right)_{t=t_{0}} \approx \frac{1}{\Lambda}\left(\frac{\partial u_{k}}{\partial t}\right)_{t=t_{0}}
$$

This assumption is justified by the fact that, according to Truesdell [3], $\partial T_{k h} / \partial x_{0 p}-\partial u_{k} / \partial x_{0 p} u_{h}$ is a smooth function of $t$-at least during the period of a fluctuation- whereas the fluid deformation varies very rapidly according to Eq. (4). This implies that the Lyapunov basis of orthonormal vectors $E_{\Lambda} \equiv\left(\mathbf{e}_{1}^{\prime}, \mathbf{e}_{2}^{\prime}, \mathbf{e}_{3}^{\prime}\right)$ [20] associated to the strain (4) rotates very quickly with respect to $\Re$ with an angular velocity $\omega_{\Lambda}$, whereas the modulus of the velocity fluctuation, measured in $E_{\Lambda}$ increases with a rate $\approx \mathrm{e}^{\Lambda\left(t-t_{0}\right)}$. Since $\Lambda$ is related to the maximal eigenvalue of $\left(\nabla \mathbf{u}+\nabla \mathbf{u}^{T}\right) / 2,\left|\boldsymbol{\omega}_{\Lambda}\right|=O(\Lambda)[1,2,21]$. Therefore, the velocity fluctuation measured in $\Re$, varies very rapidly because of the combined effect of the exponential growth rate and of the rotations of $E_{\Lambda}$ with respect to $\Re$.

Note that, since $\partial \mathbf{x} / \partial \mathbf{x}_{0}$ is supposed to vary much more fastly than the fluid state variables, as long as $t-t_{0}$ does not exceed very much the Lyapunov time $1 / \Lambda$, the Lyapunov vectors almost coincide with the eigenvectors of $\nabla \mathbf{u}$ and $\Lambda$ is nearly the maximum eigenvalue of $\left(\nabla \mathbf{u}+\nabla \mathbf{u}^{T}\right) / 2[22,23]$.

\section{Lyapunov analysis of the relative motion}

In order to investigate the mechanism of energy cascade, we study the relative motion between two fluid particles with the Lyapunov analysis.

Consider now two fixed points of the space, $\mathbf{X}$ and $\mathbf{X}^{\prime}$ (see Fig. 1) with $\mathbf{r}=\mathbf{X}^{\prime}-\mathbf{X}$, where $r=|\mathbf{r}|$ is the separation distance, and the motion of the two fluid particles which at the time $t_{0}$ pass through $\mathbf{X}$ and $\mathbf{X}^{\prime}$. The equations of motion of these particles are

$$
\frac{d \mathbf{x}}{d t}=\mathbf{u}(\mathbf{x}, t), \quad \frac{d \mathbf{x}^{\prime}}{d t}=\mathbf{u}\left(\mathbf{x}^{\prime}, t\right)
$$

where $\mathbf{u}(\mathbf{x}, t)$ and $\mathbf{u}\left(\mathbf{x}^{\prime}, t\right)$ vary with the time according to the Navier-Stokes equations. $\hat{\mathbf{r}}=\mathbf{x}^{\prime}-\mathbf{x}$ is the relative position between the two particles, therefore $\hat{\mathbf{r}}\left(t_{0}\right)=\mathbf{r}$. 


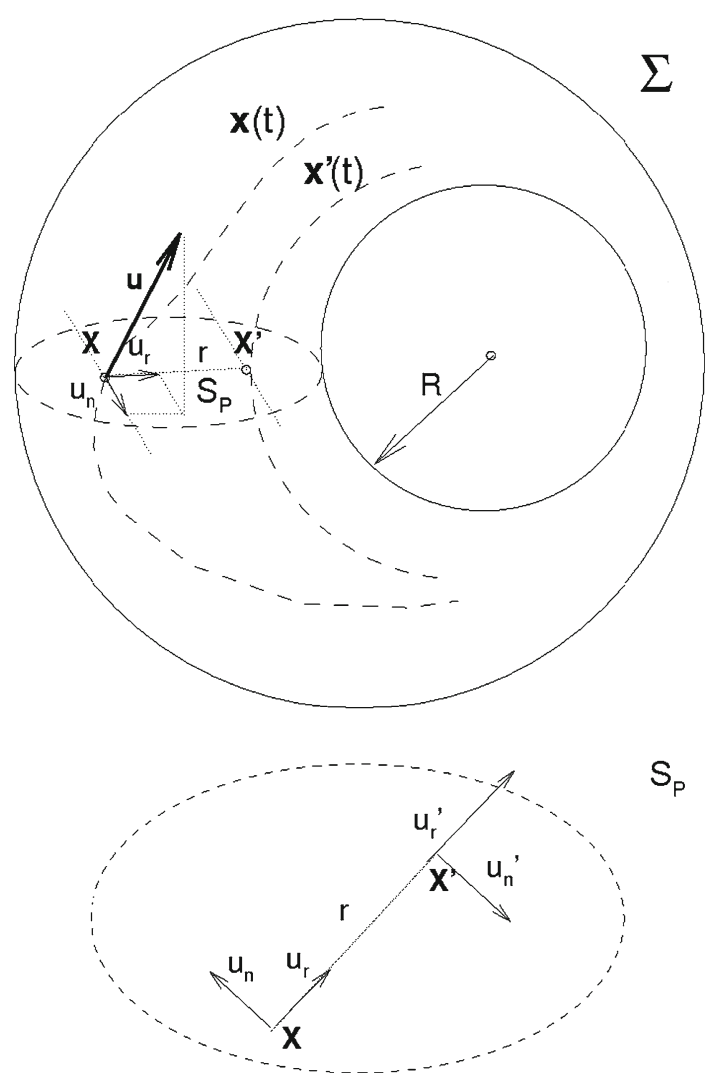

Fig. 1 Scheme of the relative motion of two fluid particles

The Lyapunov analysis of Eqs. (6) leads to the determination of the maximal finite scale Lyapunov exponent $\lambda$

$$
\lambda(r)=\lim _{T \rightarrow \infty} \frac{1}{T} \int_{0}^{T} \frac{1}{\hat{\mathbf{r}} \cdot \hat{\mathbf{r}}} \frac{d \hat{\mathbf{r}}}{d t} \cdot \hat{\mathbf{r}} d t
$$

where $\lambda(0)=\Lambda$, and $r$ represents the scale associated to $\lambda$. To define the finite scale Lyapunov basis of Eqs. (6), consider the system

$$
\begin{aligned}
& \frac{d \mathbf{x}}{d t}=\mathbf{u}(\mathbf{x}, t) \\
& \frac{d \hat{\mathbf{r}}}{d t}=\mathbf{u}(\mathbf{x}+\hat{\mathbf{r}}, t)-\mathbf{u}(\mathbf{x}, t)
\end{aligned}
$$

The finite scale Lyapunov vectors $\mathbf{r}_{1}, \mathbf{r}_{2}$ and $\mathbf{r}_{3}$ are the solutions of Eq. (9) for given initial conditions $\mathbf{r}_{i}(0)$, $(i=1,2,3)$ [18], where $\mathbf{x}$ and $\mathbf{u}$ vary according to Eq. (8) and the Navier-Stokes equations, respectively. Such vectors, also known as Bred vectors, are, by definition, related to the classical Lyapunov vectors in such a way that, $\mathbf{r}_{1}, \mathbf{r}_{2}$ and $\mathbf{r}_{3}$ tend to the classical Lyapunov vectors when $\mathbf{r}_{i}(0) \rightarrow 0,(i=1,2,3)[19,24]$. The finite scale Lyapunov basis $E_{\lambda} \equiv\left(\mathbf{e}_{1}, \mathbf{e}_{2}, \mathbf{e}_{3}\right)$ of Eqs. (6) is then obtained by orthogonalizing $\mathbf{r}_{1}, \mathbf{r}_{2}$ and $\mathbf{r}_{3}$ with the Gram-Schmidt method at each time [20,24].

This basis rotates with respect to $\Re$ with an angular velocity $-\boldsymbol{\omega}_{\lambda}$, where $\left|\boldsymbol{\omega}_{\lambda}\right| \approx \lambda(r)$ because of the isotropy. The fluid velocity difference $\Delta \mathbf{v} \equiv\left(v_{1}^{\prime}-v_{1}, v_{2}^{\prime}-v_{2}, v_{3}^{\prime}-v_{3}\right)$, measured in $E_{\lambda}$, is expressed by the Lyapunov theory as

$$
v_{l}^{\prime}-v_{l}=\lambda_{l} \hat{r}_{l}, \quad l=1,2,3
$$


Into Eq. (10), $\lambda_{l}$ is the Lyapunov exponent associated to the direction $\hat{r}_{l}$, where $\hat{r}_{l}, v_{l}$ and $v_{l}^{\prime}$ are, respectively, the components of $\mathbf{x}^{\prime}-\mathbf{x}$ and of $\mathbf{u}(\mathbf{X}, t)$ and $\mathbf{u}\left(\mathbf{X}^{\prime}, t\right)$ measured in $E_{\lambda} . \Delta \mathbf{v}$ can be also written in terms of the maximum exponent $\lambda$

$$
\Delta \mathbf{v}(\hat{\mathbf{r}})=\lambda(r) \hat{\mathbf{r}}+\zeta
$$

where $\zeta$, due to the other two exponents, makes $\Delta \mathbf{v}(r)$ a solenoidal field, being $|\zeta|<<|\lambda \mathbf{r}|$ during the fluctuation. When $\zeta / \lambda r \rightarrow 0, \hat{\mathbf{r}}$ maintains unchanged orientation with respect to $E_{\lambda}$. The velocity difference $\Delta \mathbf{u}$, measured in $\Re$ and expressed in $E_{\lambda}$, is calculated when $|\hat{\mathbf{r}}|=r$

$$
\Delta \mathbf{u}(\hat{\mathbf{r}})=\lambda(r) \hat{\mathbf{r}}+\zeta+\omega_{\lambda} \times \hat{\mathbf{r}}
$$

This equation states that $\boldsymbol{\omega}_{\lambda}$ determines the lateral components of $\Delta \mathbf{u}$ in $E_{\lambda}$.

The longitudinal component of the velocity difference in $\Re, \Delta u_{r}$ is then

$$
\Delta u_{r}(\hat{\mathbf{r}})=\boldsymbol{\xi} \cdot \mathbf{Q} \Delta \mathbf{u}(\hat{\mathbf{r}})
$$

where $\mathbf{Q} \equiv\left(\left(e_{i j}\right)\right)$ is the rotation matrix transformation from $E_{\lambda}$ to $\Re, e_{i j}$ are the components of $\mathbf{e}_{i}$ in $\Re$, and $\xi=\left(\mathbf{X}^{\prime}-\mathbf{X}\right) /\left|\mathbf{X}^{\prime}-\mathbf{X}\right|$ is the unit vector along the longitudinal direction. Observe that, $\lambda(r)$ and $\boldsymbol{\omega}_{\lambda}$ arise from the Navier-Stokes equations and that, in line with Eq. (7), $\lambda(r)$ changes with variations much slower than $\boldsymbol{\omega}_{\lambda}$ [22,23]. The simultaneous variations $\mathbf{Q}$ and $\Delta \mathbf{u}$ produce the velocity difference fluctuation observed in $\Re$, according to Eq. (13).

Although $\omega_{\lambda}$ and $\Delta \mathbf{v}$ arise from Eqs. (8) and (9), they are not directly related, therefore, we suppose here that $\boldsymbol{\omega}_{\lambda}$ is statistically orthogonal to both $\mathbf{v}$ and $\mathbf{v}^{\prime}$, with $\left\langle\boldsymbol{\omega}_{\lambda}\right\rangle=0$. This is the crucial assumption of the present work, which is justified by the condition of fully developed turbulence. This provides that

$$
\left\langle\boldsymbol{\omega}_{\lambda} v_{p}^{m} v_{q}^{\prime n}\right\rangle=0, \quad m, n>0, p, q=1,2,3
$$

where the angular brackets denote the average calculated on the statistical ensemble of all the pairs of particles which pass through $\mathbf{X}$ and $\mathbf{X}^{\prime}$. Again thanks to the fully developed turbulence, we also suppose that $\mathbf{Q}$ and $\boldsymbol{\omega}_{\lambda}$ are statistically orthogonal each other. This implies that

$$
\left\langle\omega_{\lambda p}^{m} e_{i q}^{n}\right\rangle=\left\langle\omega_{\lambda p}^{m}\right\rangle\left\langle e_{i q}^{n}\right\rangle, \quad m, n>0, i, p, q=1,2,3
$$

whereas the isotropy gives

$$
\left\langle\omega_{\lambda h} \omega_{\lambda k}\right\rangle=\frac{1}{3}\left\langle\omega_{\lambda} \cdot \omega_{\lambda}\right\rangle \delta_{h k}, \quad\left\langle e_{h p} e_{k q}\right\rangle=\frac{1}{3} \delta_{h k} \delta_{p q}
$$

where $\omega_{\lambda} \equiv\left(\omega_{\lambda 1}, \omega_{\lambda 2}, \omega_{\lambda 3}\right)$, and $\mathbf{e}_{i} \equiv\left(e_{i 1}, e_{i 2}, e_{i 3}\right), \quad(i=1,2,3)$, are expressed in $\Re$, and

$$
\left\langle\omega_{\lambda i}^{2}\right\rangle=a_{i} \lambda^{2}(r), i=1,2,3
$$

Because of homogeneity, $a_{i}=O(1)$ do not depend on $r$.

Under these hypotheses, we want to demonstrate the validity of the following equations:

$$
\begin{aligned}
\left\langle u_{r} u_{r}^{\prime} \omega_{\lambda k}\right\rangle & =0 \\
\left\langle u_{n} u_{n}^{\prime} \omega_{\lambda k}\right\rangle & =\left\langle u_{b} u_{b}^{\prime} \omega_{\lambda k}\right\rangle=C_{k} u^{2} \lambda(r) g(r)
\end{aligned}
$$

where $u_{r}, u_{n}$ and $u_{b}$ are the components of $\mathbf{u}$ along $\hat{\mathbf{r}}$ and along the two orthogonal directions $n$ and $b$, and $C_{k}$ is a proper constant of the order of unity. Due to homogeneity and isotropy, the two lateral velocity correlation functions $g(r)=\left\langle u_{n} u_{n}^{\prime}\right\rangle / u^{2}$, one measured in $E_{\lambda}$ and the other measured in $\Re$, coincide each other (see "Appendix A").

Equation (18) is the direct consequence of Eq. (14). In fact $\left\langle u_{r} u_{r}^{\prime} \boldsymbol{\omega}_{\lambda}\right\rangle \equiv\left\langle v_{r} v_{r}^{\prime} \boldsymbol{\omega}_{\lambda}\right\rangle=0$.

To demonstrate Eq. (19), observe that $u_{n}$ and $\omega_{\lambda}$ can be decomposed into $n$ independent, identically distributed, stochastic variables $\xi_{k}$, which satisfy [25]

$$
\left\langle\xi_{k}\right\rangle=0, \quad\left\langle\xi_{h} \xi_{k}\right\rangle=\delta_{h k} \quad\left\langle\xi_{h} \xi_{k} \xi_{l}\right\rangle=\varpi_{h k j} q
$$


where $\varpi_{h k j}=1$ if $h=k=j$, else $\varpi_{h k j}=0$ and $q \neq 0 . \omega_{\lambda j}$ is expressed as the sum of $\xi_{k}$, where, without loss of generality, the coefficients of the combination are assumed constant with respect to $\mathbf{X}$ and equal each other [25]

$$
\omega_{\lambda j}=A_{j} \lambda(r) \frac{1}{n} \sum_{k=1}^{n} \xi_{k}
$$

with $A_{1}=A_{2}=A_{3}=O(1)$ constant parameters. The component $u_{n}$ (and $u_{b}$ ) is also expressed as the linear combination of $\xi_{k}$, whose coefficients are now functions of $\mathbf{X}$ as the consequence of the previous assumption

$$
u_{n}(\mathbf{X})=u \sum_{k=1}^{n} F_{k}(\mathbf{X}) \xi_{k}
$$

Hence, $g(r)$ is in terms of $F_{k}$ and is determined through Eqs. (22) and (20) [25], putting $\mathbf{X}=0$

$$
g(r)=\sum_{k=1}^{n} F_{k}(0) F_{k}(\mathbf{r})
$$

and $\left\langle u_{n} u_{n}^{\prime} \omega_{\lambda k}\right\rangle$ is calculated taking into account Eqs. (21), (22), (23) and (20), and, as a result, Eq. (19) is achieved.

\section{Closure of the von Kármán-Howarth equation}

Now, we present the closure of the von Kármán-Howarth equation based on the analysis seen at the previous section.

The term representing the inertia forces in the von Kármán-Howarth equation satisfies the identity (73) (see "Appendix A"), which is here written as [6,7]

$$
\frac{\partial}{\partial r_{k}}\left(r_{k} K\right)=\frac{\partial}{\partial r_{k}}\left\langle u_{i} u_{i}^{\prime}\left(u_{k}-u_{k}^{\prime}\right)\right\rangle
$$

where, due to the isotropy, $K$ is a function of $r$ alone [6]. The divergence of $\mathbf{r} K$ gives the mechanism of the energy cascade which does not depend upon the frame of reference $[6,7]$. In order to determine the expression of $K$, Eq. (24) is here written in $E_{\lambda}$. In view of Eq. (12) and taking into account that $u_{i} u_{i}^{\prime}$ is also frame independent, one obtains the following equation

$$
\frac{\partial}{\partial \hat{r}_{k}}\left(\hat{r}_{k} K\right)=-\frac{\partial}{\partial \hat{r}_{k}}\left(\left\langle u_{i} u_{i}^{\prime} \lambda\right\rangle \hat{r}_{k}+\left(\left\langle u_{i} u_{i}^{\prime} \boldsymbol{\omega}_{\lambda}\right\rangle \times \hat{\mathbf{r}}\right)_{k}+\left\langle u_{i} u_{i}^{\prime} \zeta_{k}\right\rangle\right)
$$

Since $\lambda$ is calculated with Eq. (7), this is constant with respect to the statistics of $u_{i}$ and $u_{i}^{\prime}$, thus $\left\langle\lambda u_{i} u_{i}^{\prime}\right\rangle=$ $\lambda\left\langle u_{i} u_{i}^{\prime}\right\rangle$, and $K$ is expressed as the general integral of Eq. (25)

$$
K \hat{\mathbf{r}}=-\lambda\left\langle u_{i} u_{i}^{\prime}\right\rangle \hat{\mathbf{r}}-\left\langle u_{i} u_{i}^{\prime} \boldsymbol{\omega}_{\lambda}\right\rangle \times \hat{\mathbf{r}}+\mathbf{s}
$$

Into Eq. (26), $\mathbf{s}$ is the sum of a term due to $\zeta$ plus an arbitrary solenoidal field arising from the integration of Eq. (25) [26]. According to this analysis, $\mathbf{s}$ is proportional to $u^{2} \lambda r$ and can be written in the form

$$
\mathbf{s}=u^{2} \lambda(r) r \mathbf{s}_{0}
$$

Substituting Eq. (69) (see "Appendix A") and Eq. (27) into Eq. (26), $K \hat{\mathbf{r}}$ is

$$
K \hat{\mathbf{r}}=\left(\lambda u^{2}(g-f)-3 \lambda u^{2} g\right) \hat{\mathbf{r}}-\left\langle u_{i} u_{i}^{\prime} \boldsymbol{\omega}_{\lambda}\right\rangle \times \hat{\mathbf{r}}+u^{2} \lambda(r) r \mathbf{s}_{0}
$$

where $f(r)$ is the longitudinal velocity correlation function.

Equation (28) is made by three addends. In the first one, the part into the brackets is an even function of $r$ which goes to zero as $r \rightarrow \infty$ and assumes the value $-3 u^{2} \lambda(0)$ for $r=0$. The second term, orthogonal to 
the first one, vanishes at $r=0$ and tends to zero when $r \rightarrow \infty$. In this latter, for sake of convenience, $u_{i} u_{i}^{\prime}$ is expressed in $E_{\lambda}$

$$
\left\langle u_{i} u_{i}^{\prime} \omega_{\lambda}\right\rangle=\left\langle u_{r} u_{r}^{\prime} \omega_{\lambda}\right\rangle+\left\langle u_{n} u_{n}^{\prime} \omega_{\lambda}\right\rangle+\left\langle u_{b} u_{b}^{\prime} \omega_{\lambda}\right\rangle
$$

The first term of the RHS of Eq. (29) vanishes because of Eq. (18), whereas the other ones are expressed by means of Eq. (19)

$$
\left\langle u_{i} u_{i}^{\prime} \boldsymbol{\omega}_{\lambda}\right\rangle \times \hat{\mathbf{r}}=2 u^{2} \lambda(r) g(r) \mathbf{c} \times \hat{\mathbf{r}}
$$

where $\mathbf{c} \equiv\left(C_{1}, C_{2}, C_{3}\right)$ and $C_{k}=O(1)$ are from Eq. (19).

In order to satisfy Eq. (28), the expression of $\mathbf{s}_{0}$ must be of the kind $\mathbf{s}_{0}=h(r) \hat{\mathbf{t}}+p(r) \mathbf{n}$, where, because of homogeneity and according to Refs. [7] and [27], $h(r)$ and $p(r)$ are even functions of $r$. Moreover, the isotropy requires that $h(r)=p(r)[7,27]$, so $\mathbf{s}_{0}$ is

$$
\mathbf{s}_{0}=h(r)(\mathbf{t}+\mathbf{n})
$$

where $\mathbf{t}=\hat{\mathbf{r}} / r, \mathbf{n}=(\mathbf{c} \times \hat{\mathbf{r}}) /|\mathbf{c} \times \hat{\mathbf{r}}|$.

To determine $K$ and $h(r)$, Eq. (26) is projected along the directions $\hat{\mathbf{r}}$ and $\mathbf{n}$

$$
\begin{array}{r}
K=\lambda u^{2}(g-f)-3 u^{2} \lambda g+\frac{\mathbf{s} \cdot \hat{\mathbf{r}}}{r^{2}} \\
\mathbf{s} \cdot \mathbf{n}=\left\langle u_{i} u_{i}^{\prime} \boldsymbol{\omega}_{\lambda} \times \hat{\mathbf{r}}\right\rangle \cdot \mathbf{n}
\end{array}
$$

From Eq. (33)

$$
h(r)=H g(r)
$$

where $H=2(\mathbf{c} \times \hat{\mathbf{r}}) \cdot \mathbf{n} / r=O(1)$. As $\zeta / r \rightarrow 0, \hat{\mathbf{r}}$ maintains unchanged orientation in $E_{\lambda}$, thus $H$ is an invariant which has to be identified. The function $K$ is determined with Eq. (32)

$$
K=\lambda u^{2}(g-f)+u^{2} \lambda(r) g(r)(H-3)
$$

$K(r)$ satisfies the conditions $\partial K(0) / \partial r=0$ and $K(0)=0$ [7], which represent, respectively, the homogeneity of the flow and the condition that the inertia forces do not modify the fluid kinetic energy. The condition $g(0)=f(0)=1$ identifies $H=3$ and brings to

$$
K=\lambda u^{2}(g-f)
$$

Due to the fluid incompressibility, $f$ and $g$ are related each other through $g=f+1 / 2 \partial f / \partial r r$ (see Eq. (71), "Appendix A"), leading to the expression

$$
K=\frac{1}{2} u^{2} \frac{\partial f}{\partial r} \lambda(r) r
$$

This expression of $K$ has been obtained studying the properties of the velocity difference in $E_{\lambda}$.

Equation (37) states that, the fluid incompressibility, expressed by $g-f \neq 0$, represents a sufficient condition to state that $K \neq 0$. This latter is determined as soon as $\lambda$ is known. To calculate $\lambda$, it is convenient to express $\Delta \mathbf{u}=\mathbf{u}\left(\mathbf{x}^{\prime}, t\right)-\mathbf{u}(\mathbf{x}, t)$ in $\Re$, with $|\hat{\mathbf{r}}|=r$. Thus, $\Delta u_{r}$ is first expressed in terms of $\hat{\mathbf{r}}$ and $\Delta \mathbf{v}$ through Eq. (13) $\left(\Delta u_{r}=\xi \cdot \mathbf{Q} \Delta \mathbf{u}\right)$, then its standard deviation is calculated assuming that $\zeta=0$, (i.e. $\left.\Delta \mathbf{u}=\lambda \hat{\mathbf{r}}+\boldsymbol{\omega}_{\lambda} \times \hat{\mathbf{r}}\right)$ and taking into account that $\mathbf{Q}$ and $\boldsymbol{\omega}_{\lambda}$ satisfy Eq. (16), with $\left\langle\boldsymbol{\omega}_{\lambda}\right\rangle=0$ :

$$
\begin{aligned}
\left\langle\left(\Delta u_{r}\right)^{2}\right\rangle= & \sum_{i, j, k, l} \sum_{p, q, r, s}\left(\xi_{i} \xi_{p}\left\langle\lambda^{2} e_{i j} e_{p q}\right\rangle \hat{r}_{j} \hat{r}_{q}+\xi_{i} \xi_{p}\left\langle\lambda e_{i j} e_{p q} \omega_{\lambda r}\right\rangle \hat{r}_{j} \hat{r}_{s} \varepsilon_{q r s}\right. \\
& \left.+\xi_{i} \xi_{p}\left\langle\lambda e_{i j} e_{p q} \omega_{\lambda k}\right\rangle \hat{r}_{q} \hat{r}_{l} \varepsilon_{j k l}+\xi_{i} \xi_{p}\left\langle e_{i j} e_{p q} \omega_{\lambda k} \omega_{\lambda r}\right\rangle \hat{r}_{l} \hat{r}_{s}\right) \varepsilon_{j k l} \varepsilon_{q r s}
\end{aligned}
$$

where $\xi \equiv\left(\xi_{1}, \xi_{2}, \xi_{3}\right)$ and $\varepsilon_{i j k}=(j-i)(k-i)(k-j) / 2$ represents the Levi-Civita tensor arising from the cross product.

Since $\lambda$ is the average of the velocity increment per unit distance, it is constant with respect the statistics of $\mathbf{Q}$ and $\omega_{\lambda}$, thus $\left\langle\lambda^{2} \ldots\right\rangle=\lambda^{2}\langle\ldots\rangle$. Because of Eq. (15), $\mathbf{Q}$ and $\omega_{\lambda}$ are statistically independent, so that $\left\langle e_{i j} e_{p q} \omega_{\lambda k}\right\rangle=\left\langle e_{i j} e_{p q}\right\rangle\left\langle\omega_{\lambda k}\right\rangle=0$ and $\left\langle e_{i j} e_{p q} \omega_{\lambda k} \omega_{\lambda r}\right\rangle=\left\langle e_{i j} e_{p q}\right\rangle\left\langle\omega_{\lambda k} \omega_{\lambda r}\right\rangle$, therefore second and third terms vanish into Eq. (38). 
Due to the isotropy, the Lyapunov basis satisfies Eq. (16) $\left(\left\langle e_{i j} e_{p q}\right\rangle=\delta_{i p} \delta_{j q} / 3\right)$. This is introduced in the first term of Eq. (38), which thus depends on $r^{2}$ alone. This term, which equals $\lambda^{2} r^{2} / 3$, is associated to the direction $\hat{\mathbf{r}}$ (maximal Lyapunov exponent direction) and represents one degree of freedom in the space. Again thanks to the isotropy, the last term of Eq. (38) is twice the first one because it is relative to the two directions orthogonal to $\hat{\mathbf{r}}$ and thus to two degrees of freedom. As a result, taking into account that $\varepsilon_{i j k} \varepsilon_{i j h}=2 \delta_{h k}$, the standard deviation of the longitudinal velocity difference is

$$
\left\langle\left(\Delta u_{r}\right)^{2}\right\rangle=\lambda^{2} r^{2}
$$

with

$$
\lambda(r)=\sqrt{\frac{\left\langle\omega_{\lambda} \cdot \omega_{\lambda}\right\rangle}{3}}
$$

This standard deviation can be expressed through the longitudinal correlation function $f$

$$
\left\langle\left(\Delta u_{r}\right)^{2}\right\rangle=2 u^{2}(1-f(r))
$$

being $u$ the standard deviation of the longitudinal velocity. The maximal Lyapunov exponent is calculated in function of $f$, from Eqs. (39) and (41)

$$
\lambda(r)=\frac{u}{r} \sqrt{2(1-f(r))}
$$

Hence, substituting Eq. (42) into Eq. (37), one obtains the expression of $K$ in terms of $f$ and its gradient

$$
K=u^{3} \sqrt{\frac{1-f}{2}} \frac{\partial f}{\partial r}
$$

This is the proposed closure of the von Kármán-Howarth equation. Its main asset with respect to the other diffusion models is that it has been derived from a specific Lyapunov analysis, under the assumption of reasonable statistical hypotheses about the longitudinal velocity difference. According to Eq. (43), $K$ does not represent a diffusion approximation, but rather a nonlinear advection term which makes Eq. (72) a nonlinear partial differential equation of the first order when $v=0$.

Equation (43) corresponds to a mechanism of the kinetic energy transfer which preserves the average values of the momentum and of the kinetic energy, and satisfies the continuity equation. Specifically, the analytical structure of Eq. (43) states that this mechanism consists of a flow of the kinetic energy from large to small scales which only redistributes the kinetic energy between wavelengths.

This mechanism can be interpreted as follows. If, at $t_{0}$, a toroidal volume $\Sigma\left(t_{0}\right)$ is taken which contains $\mathbf{X}$ and $\mathbf{X}^{\prime}$ (see Fig. 1), its geometry and position change according to the fluid motion, and its dimensions, $\sqrt{S_{p}}$ and $R$, vary with the time to preserve the volume. Choosing $\Sigma$ in such a way that $R$ increases with the time, the Lyapunov analysis of Eqs. (6) leads to $R \approx R\left(t_{0}\right) \mathrm{e}^{\lambda\left(t-t_{0}\right)}$. According to the theory [22], for $t>t_{0}$, the trajectories of the two particles are enclosed into $\Sigma(t)$. Hence, the kinetic energy, initially enclosed into $\Sigma\left(t_{0}\right)$, at the end of the fluctuation is contained into $\Sigma(t)$ whose dimensions are changed with respect to $\Sigma\left(t_{0}\right)$. The kinetic energy is then transferred far from $\mathbf{X}$ and $\mathbf{X}^{\prime}$, resulting enclosed in a more thin toroid.

\section{Skewness of the velocity difference PDF}

The obtained expression of $K(r)$ allows to determine the skewness of $\Delta u_{r}$ [7]

$$
H_{3}(r)=\frac{\left\langle\left(\Delta u_{r}\right)^{3}\right\rangle}{\left\langle\left(\Delta u_{r}\right)^{2}\right\rangle^{3 / 2}}=\frac{6 k(r)}{(2(1-f(r)))^{3 / 2}}
$$

which is expressed in terms of the longitudinal triple correlation $k(r)$, linked to $K(r)$ by $K(r)=$ $u^{3}(\partial / \partial r+4 / r) k(r)$ (also see "Appendix A", Eq. (74)). Since $f$ and $k$ are, respectively, even and odd functions of $r$ with $f(0)=1, k(0)=k^{\prime}(0)=k^{\prime \prime}(0)=0, H_{3}(0)$ is given by

$$
H_{3}(0)=\lim _{r \rightarrow 0} H_{3}(r)=\frac{k^{\prime \prime \prime}(0)}{\left(-f^{\prime \prime}(0)\right)^{3 / 2}}
$$

where the apex denote the derivative with respect to $r$. To obtain $H_{3}(0)$, observe that, near the origin 


$$
K=u^{3} \sqrt{-f^{\prime \prime}(0)} f^{\prime \prime}(0) \frac{r^{2}}{2}+O\left(r^{4}\right)
$$

then, substituting Eq. (46) into $K(r)=u^{3}(\partial / \partial r+4 / r) k(r)$ and accounting for Eq. (45), one obtains

$$
H_{3}(0)=-\frac{3}{7}=-0.42857 \ldots
$$

$H_{3}(0)$ is a constant of the present analysis, which does not depend on the Reynolds number. This is in agreement with the several sources of data existing in the literature such as [7,28-30] (and Refs. therein) and its value gives the entity of the mechanism of the energy cascade.

The skewness causes variations in the time of the Taylor scale in accordance to Eq. (72). The time derivative of $\lambda_{T}$ is calculated considering the coefficients of the order $O\left(r^{2}\right)$ in the von Kármán-Howarth equation [6,7] which are determined substituting Eqs. (46) and (47) into Eq. (72)

$$
\frac{d \lambda_{T}}{d t}=-\frac{u}{2}+v\left(\frac{7}{3} f^{I V}(0) \lambda_{T}^{3}-\frac{5}{\lambda_{T}}\right)
$$

where $f^{I V} \equiv \partial^{4} f / \partial r^{4}$. The first term tends to decrease $\lambda_{T}$ and expresses the mechanism of energy cascade, whereas the term in the brackets gives the viscosity effect which, in general, tends to increase $\lambda_{T}$ depending on the current values of $f^{I V}(0)$ and $\lambda_{T}[6,7]$.

\section{Statistical analysis of the velocity difference}

As explained in this section, the Lyapunov analysis of the local deformation and some plausible assumptions about the statistics of the velocity difference $\Delta \mathbf{u}(\mathbf{r}) \equiv \mathbf{u}(\mathbf{X}+\mathbf{r})-\mathbf{u}(\mathbf{X})$ lead to determine all the statistical moments of $\Delta \mathbf{u}(\mathbf{r})$ with the only knowledge of the function $K(r)$ and of the value of the critical Reynolds number.

The statistical properties of $\Delta \mathbf{u}(\mathbf{r})$, are investigated expressing the velocity fluctuation, given by Eq. (5), as the Fourier series

$$
\mathbf{u} \approx \frac{1}{\Lambda} \sum_{\boldsymbol{\kappa}} \frac{\partial \mathbf{U}}{\partial t}(\boldsymbol{\kappa}) \mathrm{e}^{i \boldsymbol{\kappa} \cdot \mathbf{x}}
$$

where $\mathbf{U}(\boldsymbol{\kappa}) \equiv\left(U_{1}(\boldsymbol{\kappa}), U_{2}(\boldsymbol{\kappa}), U_{3}(\boldsymbol{\kappa})\right)$ are the components of the velocity spectrum, which satisfy the Fourier transformed Navier-Stokes equations [7]

$$
\frac{\partial U_{p}(\boldsymbol{\kappa})}{\partial t}=-v k^{2} U_{p}(\boldsymbol{\kappa})+i \sum_{\mathbf{j}}\left(\frac{\kappa_{p} \kappa_{q} \kappa_{r}}{\kappa^{2}} U_{q}(\mathbf{j}) U_{r}(\boldsymbol{\kappa}-\mathbf{j})-\kappa_{q} U_{q}(\mathbf{j}) U_{p}(\boldsymbol{\kappa}-\mathbf{j})\right)
$$

All the components $\mathbf{U}(\boldsymbol{\kappa}) \approx \partial \mathbf{U}(\boldsymbol{\kappa}) / \partial t / \Lambda$ are random variables distributed according to certain distribution functions, which are statistically orthogonal each other [7].

Thanks to the local isotropy, $\mathbf{u}$ is sum of several dependent random variables which are identically distributed [7], therefore $\mathbf{u}$ tends to a gaussian variable [25], and $\mathbf{U}(\boldsymbol{\kappa})$ satisfies the Lindeberg condition, a very general necessary and sufficient condition for satisfying the central limit theorem [25]. This condition does not apply to the Fourier coefficients of $\Delta \mathbf{u}$. In fact, since $\Delta \mathbf{u}$ is the difference between two dependent gaussian variables, its PDF could be a non-gaussian distribution function. In $\mathbf{x}=0$, the velocity difference $\Delta \mathbf{u}(\mathbf{r}) \equiv\left(\Delta u_{1}, \Delta u_{2}, \Delta u_{3}\right)$ is given by

$$
\Delta u_{p} \approx \frac{1}{\Lambda} \sum_{\boldsymbol{\kappa}} \frac{\partial U_{p}(\boldsymbol{\kappa})}{\partial t}\left(\mathrm{e}^{i \boldsymbol{\kappa} \cdot \mathbf{r}}-1\right) \equiv L+B+P+N
$$

This fluctuation consists of the contributions appearing into Eq. (50): in particular, $L$ represents the sum of all linear terms due to the viscosity and $B$ is the sum of all bilinear terms arising from inertia and pressure forces. $P$ and $N$ are, respectively, the sums of definite positive and negative square terms, which derive from inertia and pressure forces. The quantity $L+B$ tends to a gaussian random variable being the sum of statistically 
orthogonal terms [31,25], while $P$ and $N$ do not, as they are linear combinations of squares [31]. Their general expressions are [31]

$$
\begin{aligned}
P & =P_{0}+\eta_{1}+\eta_{2}^{2} \\
N & =N_{0}+\zeta_{1}-\zeta_{2}^{2}
\end{aligned}
$$

where $P_{0}$ and $N_{0}$ are constants, and $\eta_{1}, \eta_{2}, \zeta_{1}$ and $\zeta_{2}$ are four different centered random gaussian variables. Therefore, the fluctuation of the longitudinal velocity difference can be written as

$$
\Delta u_{r}=\psi_{1}(\mathbf{r}) \xi+\psi_{2}(\mathbf{r})\left(\chi\left(\eta^{2}-1\right)-\left(\zeta^{2}-1\right)\right)
$$

where $\xi, \eta$ and $\zeta$ are independent centered random variables which have gaussian distribution functions with standard deviation equal to the unity. The parameter $\chi$ is a positive definite function of the Reynolds number, whereas $\psi_{1}$ and $\psi_{2}$ are functions of the space coordinates and the Reynolds number.

At the Kolmogorov scale $\ell$, the order of magnitude of the velocity fluctuations is $u_{K}{ }^{2} \tau / \ell$, with $\tau=1 / \Lambda$ and $u_{K}=v / \ell$, whereas $\psi_{2}$ is negligible because is due to the inertia forces: this immediately identifies $\psi_{1} \approx u_{K}^{2} \tau / \ell$.

On the contrary, at the Taylor scale $\lambda_{T}, \psi_{1}$ is negligible and the order of magnitude of the velocity fluctuations is $u^{2} \tau / \lambda_{T}$, therefore $\psi_{2} \approx u^{2} \tau / \lambda_{T}$.

The ratio $\psi_{2} / \psi_{1}$ is a function of $R_{\lambda}$

$$
\psi\left(\mathbf{r}, R_{\lambda}\right)=\frac{\psi_{2}(\mathbf{r})}{\psi_{1}(\mathbf{r})} \approx \frac{u^{2} \ell}{u_{K}{ }^{2} \lambda_{T}}=\sqrt{\frac{R_{\lambda}}{15 \sqrt{15}}} \hat{\psi}(\mathbf{r})
$$

where $\hat{\psi}(\mathbf{r})=O(1)$, is a function which has to be determined.

Hence, the dimensionless longitudinal velocity difference is written as

$$
\frac{\Delta u_{r}}{\sqrt{\left\langle\left(\Delta u_{r}\right)^{2}\right\rangle}}=\frac{\xi+\psi\left(\chi\left(\eta^{2}-1\right)-\left(\zeta^{2}-1\right)\right)}{\sqrt{1+2 \psi^{2}\left(1+\chi^{2}\right)}}
$$

and the dimensionless statistical moments of $\Delta u_{r}$ are easily calculated considering that $\xi, \eta$ and $\zeta$ are independent gaussian variables

$$
H_{n} \equiv \frac{\left\langle\left(\Delta u_{r}\right)^{n}\right\rangle}{\left\langle\left(\Delta u_{r}\right)^{2}\right\rangle^{n / 2}}=\frac{1}{\left(1+2 \psi^{2}\left(1+\chi^{2}\right)\right)^{n / 2}} \sum_{k=0}^{n}\left(\begin{array}{l}
n \\
k
\end{array}\right) \psi^{k}\left\langle\xi^{n-k}\right\rangle\left\langle\left(\chi\left(\eta^{2}-1\right)-\left(\zeta^{2}-1\right)\right)^{k}\right\rangle
$$

where

$$
\begin{array}{r}
\left\langle\left(\chi\left(\eta^{2}-1\right)-\left(\zeta^{2}-1\right)\right)^{k}\right\rangle=\sum_{i=0}^{k}\left(\begin{array}{c}
k \\
i
\end{array}\right)(-\chi)^{i}\left\langle\left(\zeta^{2}-1\right)^{i}\right\rangle\left\langle\left(\eta^{2}-1\right)^{k-i}\right\rangle \\
\quad \times\left\langle\left(\eta^{2}-1\right)^{i}\right\rangle=\sum_{l=0}^{i}\left(\begin{array}{l}
i \\
l
\end{array}\right)(-1)^{l}\left\langle\eta^{2(i-l)}\right\rangle
\end{array}
$$

In particular, the third moment or skewness, $H_{3}$, which is responsible for the energy cascade, is

$$
H_{3}=\frac{8 \psi^{3}\left(\chi^{3}-1\right)}{\left(1+2 \psi^{2}\left(1+\chi^{2}\right)\right)^{3 / 2}}
$$

For $\chi \neq 1$, the skewness and all the odd order moments are different from zero, and for $n>3$, all the absolute moments are rising functions of $R_{\lambda}$, thus $\Delta u_{r}$ exhibits an intermittency whose entity increases with the Reynolds number. 


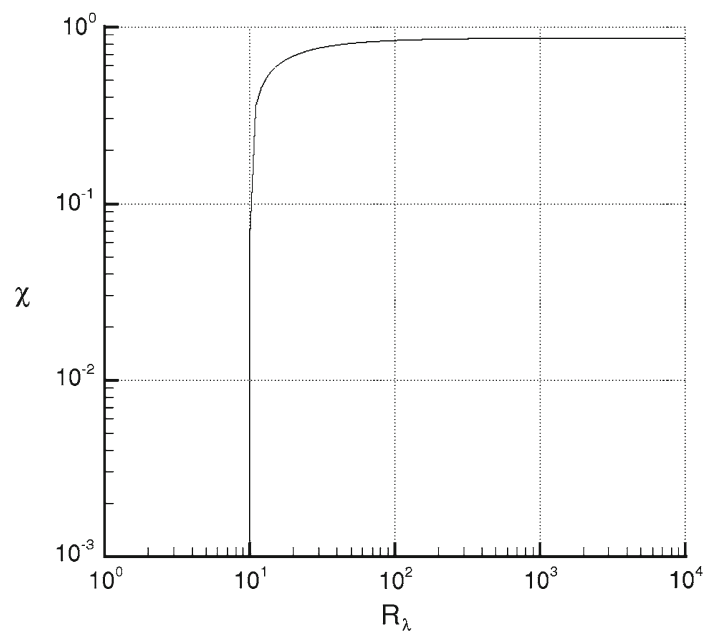

Fig. 2 Parameter $\chi$ plotted as the function of $R_{\lambda}$

All the statistical moments can be calculated once the function $\chi\left(R_{\lambda}\right)$ and the value of $\hat{\psi}_{0}$ are known. The expression of $K(r)$ obtained in the first part of the work allows to identify $H_{3}(0)$ and then fixes the relationship between $\psi_{0}$ and $\chi\left(R_{\lambda}\right)$

$$
-H_{3}(0)=\frac{8 \psi_{0}^{3}\left(1-\chi^{3}\right)}{\left(1+2 \psi_{0}^{2}\left(1+\chi^{2}\right)\right)^{3 / 2}}=\frac{3}{7}
$$

where $\psi_{0}=\psi\left(0, R_{\lambda}\right)=O\left(\sqrt{R_{\lambda}}\right)$ and $\chi=\chi\left(R_{\lambda}\right)>0$. This relationship does not admit solutions with $\chi>0$ below a minimum value of $\left(R_{\lambda}\right)_{\text {min }}$ dependent on $\hat{\psi}_{0}$. According to the analysis of "Appendix B", $\left(R_{\lambda}\right)_{\text {min }}$ is chosen to 10.12, which corresponds to $\hat{\psi}_{0} \simeq 1$.075. (setting $\chi=0, R_{\lambda}=10.12$ in $H_{3}(0)$ ). The variation of $\left(R_{\lambda}\right)_{\min }$ from 8.5 to 15 brings values of $\hat{\psi}_{0}$ between 1.2 and 0.9 , respectively. In Fig. 2 , the function $\chi\left(R_{\lambda}\right)$ is shown for $\hat{\psi}_{0}=1.075$. The limit $\chi \simeq 0.86592$ for $R_{\lambda} \rightarrow \infty$ is reached independently of the value of $\hat{\psi}_{0}$.

The PDF of $\Delta u_{r}$ is expressed through the Frobenius-Perron equation

$$
F\left(\Delta u_{r}^{\prime}\right)=\int_{\xi} \int_{\eta} \int_{\zeta} p(\xi) p(\eta) p(\zeta) \delta\left(\Delta u_{r}^{\prime}-\Delta u_{r}(\xi, \eta, \zeta)\right) d \xi d \eta d \zeta
$$

where $\Delta u_{r}$ is calculated with Eq. (55), $\delta$ is the Dirac delta and $p$ is a gaussian PDF whose average value and standard deviation are equal to 0 and 1 , respectively.

For non-isotropic turbulence or in more complex cases with boundary conditions, the velocity spectrum could not satisfy the Lindeberg condition, thus the velocity will be not distributed following a Gaussian PDF, and Eq. (53) changes its analytical form and can incorporate more intermittent terms [25] which give the deviation with respect to the isotropic turbulence. Hence, the absolute statistical moments of $\Delta u_{r}$ will be greater than those calculated with Eq. (55), indicating that, in a more complex situation than the isotropic turbulence, the intermittency of $\Delta u_{r}$ can be significantly stronger.

\section{Results and discussion}

In order to show the validity of the proposed Lyapunov analysis, several results are now presented.

As first result, we calculate the evolution in the time of $f$ with the proposed closure (Eq. (43)) of the von Kármán-Howarth equation, whose boundary conditions are Eq. (75) (see "Appendix A"). The turbulent kinetic energy, $E(\kappa)$ and $T(\kappa)$ are calculated with Eqs. (76) and (77). The calculation is carried out for an initial Reynolds number $\operatorname{Re}(0)=u(0) L_{r} / v=2000$, where $L_{r}$ and $u(0)$ are, respectively, the reference dimension and the initial velocity standard deviation. The initial condition for $f$ is $f(r)=\exp \left(-1 / 2\left(r / \lambda_{T}\right)^{2}\right)$, where $\lambda_{T} / L_{r}=1 /(2 \sqrt{2})$ and $u(0)=1$. The dimensionless time is defined as $\bar{t}=t u(0) / L_{r}$. 
Equation (72) was numerically solved adopting the Crank-Nicholson integrator scheme with variable time step $\Delta t$, whereas the discretization in the spatial domain is made by $N-1$ intervals of the same amplitude $\Delta r$. The truncation error of the scheme of integration is $\mathcal{O}\left(\Delta t^{2}\right)+\mathcal{O}\left(\Delta r^{2}\right)$, where $\Delta t$ is automatically selected by the algorithm in such a way that $\mathcal{O}\left(\Delta t^{2}\right) \sim \mathcal{O}\left(\Delta r^{2}\right)$ for each time step, thus the accuracy of the scheme is of the order of $\mathcal{O}\left(\Delta r^{2}\right)$. As the consequence, the discretization of the Fourier space is made by $N-1$ subsets in the interval $\left[0, \kappa_{M}\right]$, where $\kappa_{M}=\pi /(2 \Delta r)$. For the adopted initial Reynolds number, the choice $N=1500$ gives an adequate discretization, which provides $\Delta r<\ell$, for the whole simulation. $u$ was calculated with Eq. (76) and the kinetic energy was checked to be equal to the integral over $\kappa$ of $E(\kappa)$. During the simulation, $T(\kappa)$ must identically satisfy Eq. (78) (see "Appendix A") which states that $T(\kappa)$ does not modify the kinetic energy. $\int_{0}^{\infty} T(\kappa) d \kappa$ is calculated with the trapezoidal formula, for $\kappa \in\left(0, \kappa_{M}\right)$, thus the simulation will be considered to be accurate as long as

$$
\int_{0}^{\kappa_{M}} T(\kappa) d \kappa \simeq \int_{0}^{\infty} T(\kappa) d \kappa=0
$$

namely, when $T(\kappa) \simeq 0$ for $\kappa>\kappa_{M}$. As the simulation advances, according to Eq. (43), the energy cascade determines variations of $E(\kappa)$ and $T(\kappa)$ for values of $\kappa$ rising with the time and that can occur out of the interval $\left(0, \kappa_{M}\right)$. Thus, Eq. (61) holds until a certain time, where these wave-numbers are about equal to $\kappa_{M}$. For greater times, $T(\kappa) \neq 0$ for $\kappa>\kappa_{M}$ and Eq. (61) could not be satisfied. Hence, the simulation will be accurate until reaching the following condition [32]

$$
\left|\int_{0}^{\kappa_{M}} T(\kappa) d \kappa\right| \geq \frac{1}{12} \frac{\kappa_{M}^{3}}{N^{2}}\left|\frac{\partial^{2} T}{\partial \kappa^{2}}\right|_{\max } \approx \frac{1}{N^{2}} \int_{0}^{\kappa_{M}}|T(\kappa)| d \kappa
$$

where the right-hand side represents the estimation of the truncation error of the trapezoid formula [32]. There, it is found that, $\Delta r \approx 0.8 \mathrm{\ell}$ in all the simulations. Thereafter, the numerical method loses its consistency, since the violation of Eq. (61) implies that $K$ does not preserve the kinetic energy. Nevertheless, the error committed by the algorithm is considered still acceptable as long as $[6,7]$

$$
0<\left|\int_{0}^{\kappa_{M}} T(\kappa) d \kappa\right|<<<\left|\frac{d u^{2}}{d t}\right|
$$

Namely, when the residual of the rate of energy transfer is much less than the dissipation rate. In any case, the simulations are stopped if $\ell$ reaches its relative minimum in function of the time with $\ell>\Delta r$, or as soon as $\Delta r=\ell$. The accuracy of the solutions is evaluated through the check of Eq. (63). At the end of simulation, we assume that the energy spectrum is fully developed.

To study the proposed closure, we first analyze the case with $K=0$ with the previous initial condition. In this case, Eq. (72) admits the analytical self-similar solution [6]

$$
f(t, r)=\exp \left(-\frac{1}{2}\left(\frac{r}{\lambda_{T}(t)}\right)^{2}\right), \text { where } \lambda_{T}(t)=\sqrt{\lambda_{T}^{2}(0)+4 v t}
$$

that is, $f(t, r)$ maintains its shape unchanged in terms of the dimensionless coordinate $r / \lambda_{T}(t)$. The corresponding numerical solutions were calculated for different spatial discretization $(N=500,1000$, and 1500$)$. All these simulations satisfy Eq. (64), with a calculated error which is always of the order of the truncation error of the scheme of integration. Also, the energy spectrum preserves its shape and is given by $E\left(\kappa \lambda_{T}\right)=$ $A u^{2}\left(\kappa \lambda_{T}\right)^{4} \exp \left(-a\left(\kappa \lambda_{T}\right)^{2}\right)$, with $A=O(1), a=O(1)$ [7].

Consider now the case, where $K$ is given by Eq. (43). The diagrams in Fig. 3 show $f(r)$ and $k(r)$ in terms of $r / \lambda_{T}$, at different times of simulation. The kinetic energy and $\lambda_{T}$ vary according to Eqs. (43), (76) and (48), thus $f(r)$ and $k(r)$ change in such a way that their scales, in particular $\ell$ and $\lambda_{T}$, diminish with the time, whereas the maximum of $|k|$ decreases. Namely, Eq. (43) corresponds to a transferring of the energy toward the smaller scales which strongly contrasts the effects of viscosity seen in the previous case.

At the final instants, $f-1=\mathrm{O}\left(r^{2 / 3}\right)$ for $r / \lambda_{T}=\mathrm{O}(1)$, and the maximum of $|k|$ is about 0.05 . These results are in very good agreement with the numerous data of the literature concerning the evolution of $f$ [7]. 

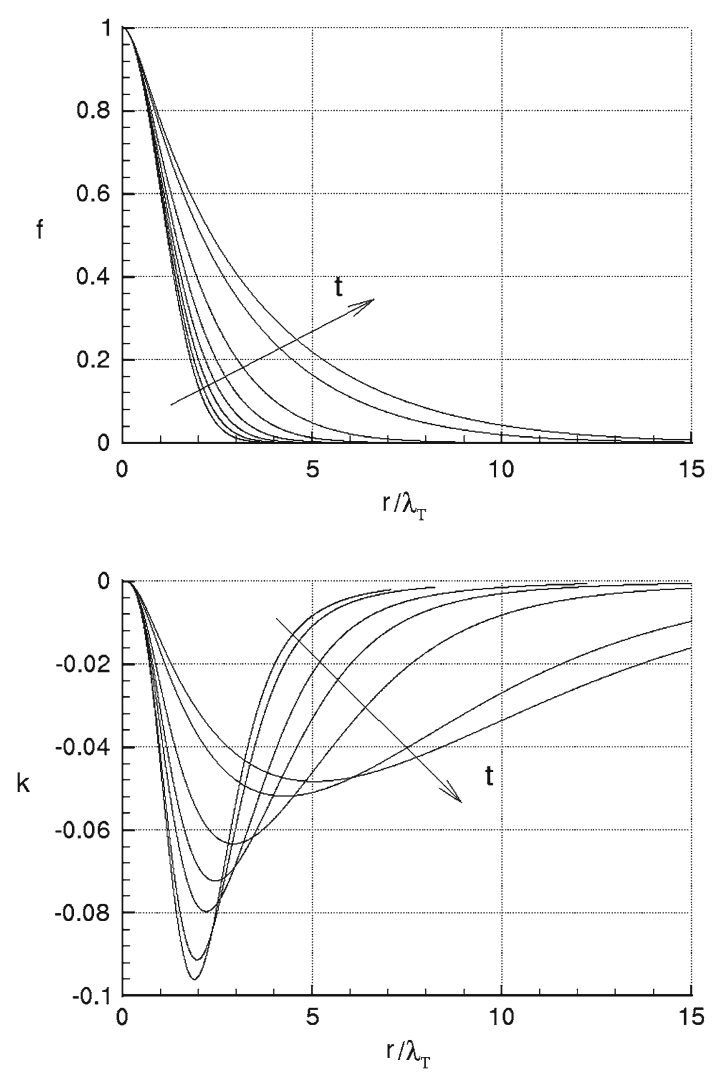

Fig. 3 Correlation functions, $f$ and $k$ versus the separation distance at the times of simulation $\bar{t}=0,0.1,0.2,0.3,0.4,0.5,0.6$, $0.63, \operatorname{Re}(0)=2,000$

Figure 4 shows $E(\kappa)$ and $T(\kappa)$, for the same times, with the Kolmogorov law $\left(\kappa^{-5 / 3}\right)$ and the incompressibility condition $\left(\kappa^{4}\right)$. The energy spectrum depends on the initial condition, and at the end of simulation, can be compared with the Kolmogorov spectrum in an opportune interval of wave-numbers which defines the inertial subrange. Furthermore, according to the proposed closure, $E(\kappa)$ satisfies the continuity equation $\left(E(\kappa) \approx \kappa^{4}\right.$ for $\kappa \lambda_{T}<<1$ ). In the figure, the dimensionless time $\bar{t}=0.63$ corresponds to the condition (62), whereas $\bar{t}=0.69$ (bold dash dotted line) represents the spectrum calculated when $\ell$ reaches its minimum in function of the time. In this last situation, the algorithm is less accurate, due to the discretization. In fact, $T(\kappa)$, (bold dash dotted line), exhibits small non-zero values in a wide range of variations of $\kappa$, including $\kappa>\kappa_{M}$. Although Eq. (61) is not satisfied, the error of the algorithm is still acceptable since $\left|\int_{0}^{\kappa_{M}} T(\kappa) d \kappa\right| /\left|d u^{2} / d t\right| \approx 10^{-4}$.

To study the effect of the spatial discretization on the final energy spectrum, Fig. 5 shows the results of simulations performed for $N=500,1,000$. It is found that, for $N=500$ and 1000 , the stopping criterion is satisfied for $\ell=\Delta r$, whereas the final times of simulation rises with $N$, resulting $\bar{t} \approx 0.33$ and $\bar{t} \approx 0.64$ for $N=500$ and 1000, respectively. Accordingly, the inertial range of Kolmogorov and $\bar{t}$ increase with $N$ until about $N=1,100$, thereafter they are about constant.

To compare the present analysis with the data in the literature, a further calculation has been carried out with an initial Reynolds number of $\operatorname{Re}(0)=3,000$. The present results (continuous line) are shown in Fig. 6 in terms of energy spectrum, and are compared with those calculated, for the same conditions, with the Oberlack's model [11] (dashed line). For this latter, the parameter $k_{2}$ [11] (see introduction) is assumed to be equal to $k_{2}=-H_{3}(0) /(\sqrt{2} 6)$. For this value of $k_{2}$, the Oberlack's model gives the same value of skewness $H_{3}(0)=-3 / 7$, calculated with the present analysis. Thus, the entity of the mechanism of the energy cascade is the same in both the cases. Again, the initial correlation function is $f=\exp \left(-\left(r / \lambda_{T}\right)^{2} / 2\right), \lambda_{T} / L_{r}=1 /(2 \sqrt{2})$ and $N=1,500$.

Equation $(61)$ is satisfied until a certain time (i.e. $t \approx 0.66$ for Oberlack's model and $t \approx 0.62$ for the present analysis), thereafter the numerical scheme exhibits a minor accuracy, since $T(\kappa) \neq 0$ for $\kappa>\kappa_{M}$. In the figure, the energy spectrum is shown at the end of simulation which happens for $\bar{t}=0.71$ (Oberlack) 

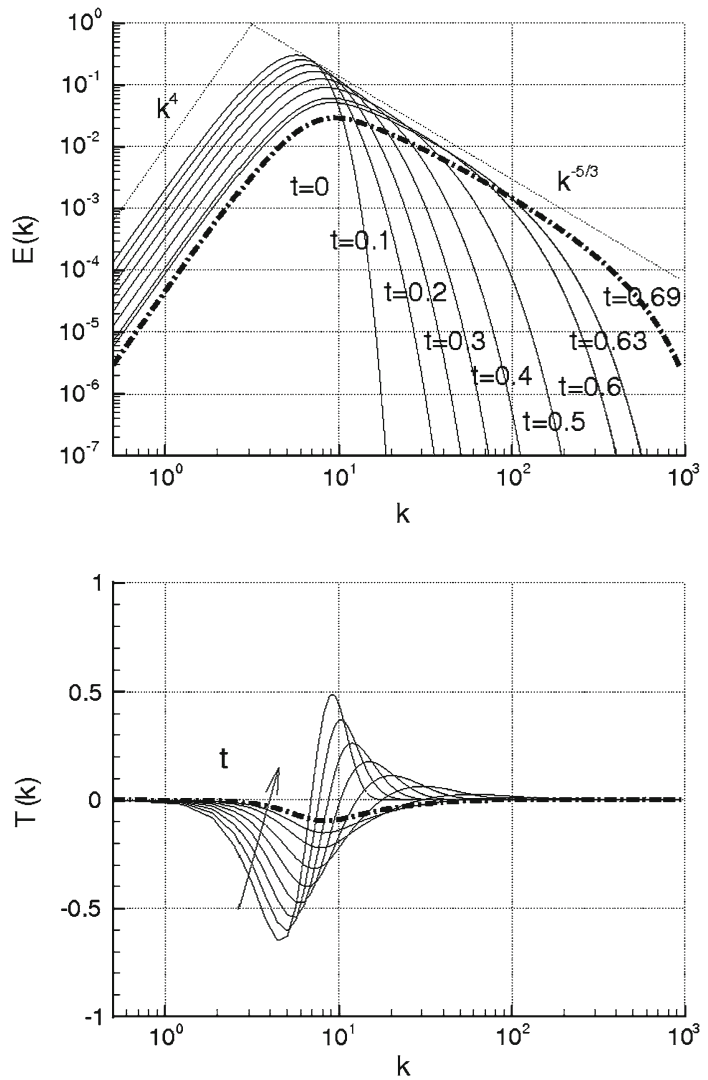

Fig. 4 Plot of $E(\kappa)$ and $T(\kappa)$, for $\operatorname{Re}(0)=2,000$, at the diverse times of simulation

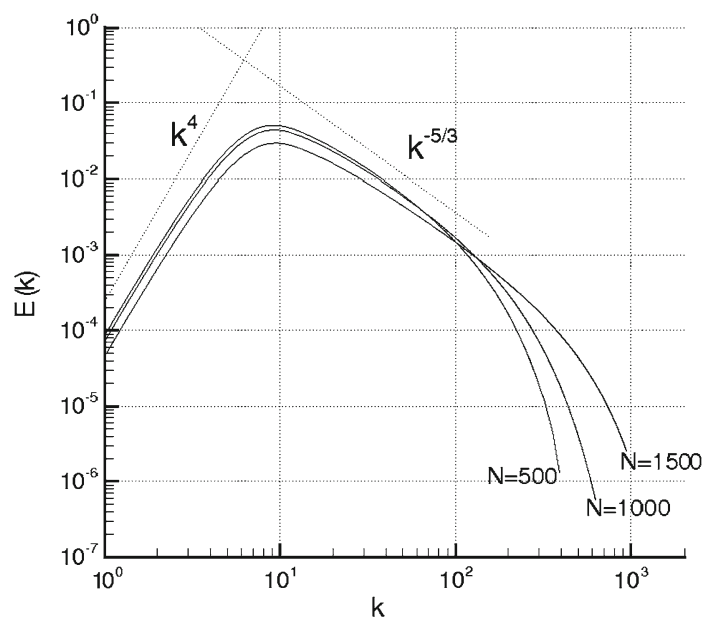

Fig. 5 Plot of the final energy spectrum for different spatial discretization, $\operatorname{Re}(0)=2,000$

and for $\bar{t}=0.67$ (Present Analysis), where the stopping condition is satisfied for $\ell=\min \approx 0.92 \Delta r$. There, $\left|\int_{0}^{\kappa M} T(\kappa) d \kappa\right| /\left|d u^{2} / d t\right| \approx 10^{-4}$, in both the cases, therefore the two simulations can be considered accurate enough. Since $\mathrm{H}_{3}(0)$ is the same in the two cases, the two values of $\lambda_{T}$ almost coincide during the simulations, a part very small variations which are not appreciated in the figure. Therefore, also $d u^{2} / d t$ is about the same in both the cases. Nevertheless, the energy spectra show significative differences. The spectrum calculated with Eq. (43) exhibits a wider range of wave-numbers than the other one, and this is due to Eq. (43) representing a nonlinear term of the first order which does not cause any diffusion effect, whereas for the Oberlack's model, the closure term of the second order and the variable eddy diffusivity, produce a sizable reduction of all the 

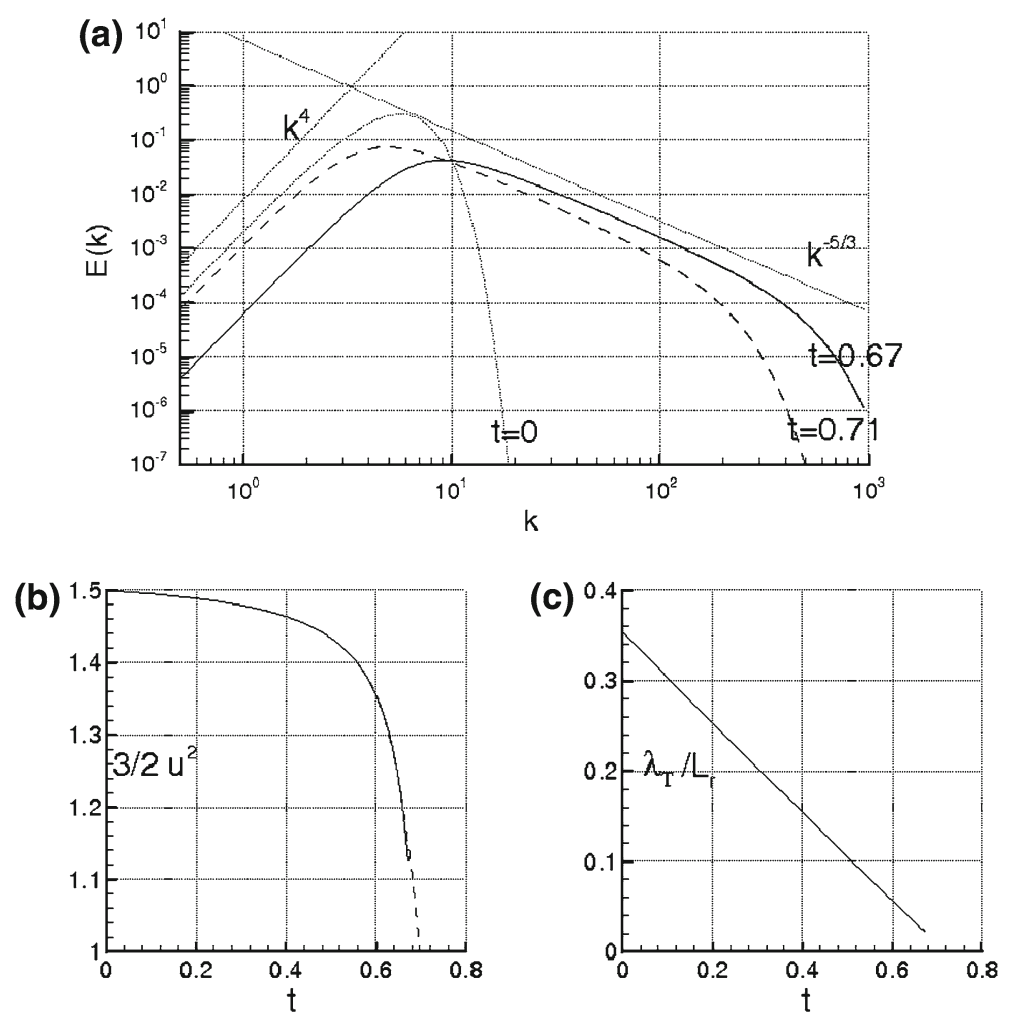

Fig. 6 Comparison of the results for $\operatorname{Re}(0)=3,000$ : Continuous line: present analysis. Dashed line: Oberlack's model (Ref. [11]). a Dotted curve: initial condition. b Kinetic energy, c Taylor scale

wave-numbers, especially for large $\kappa$. The wave-number at which $E(\kappa)=10^{-6}$ (see figure), is quite different in the two cases, being the first value almost two times the second one. Therefore, the Kolmogorov inertial subrange calculated with the present analysis is about two times that obtained with the Oberlack's model.

Next, the Kolmogorov function $Q(r)$ and Kolmogorov constant $C$, are determined, using the results of the previous simulations.

Following the Kolmogorov theory, the Kolmogorov function, defined as

$$
Q(r)=-\frac{\left\langle\left(\Delta u_{r}\right)^{3}\right\rangle}{r \varepsilon}
$$

is constant and is equal to $4 / 5$ as long as $r / \lambda_{T}=O(1)$. As the consequence of the initial correlation function (see Fig. 7), for $\bar{t}=0, Q_{\max }>>4 / 5$ and the variations of $Q$ with $r / \lambda_{T}$ cannot be neglected. For $t>0, Q_{\max }$ decreases until the final instants, where $Q(r)$ exhibits smaller variations for $r / \lambda_{T} \neq 0$ and $Q_{\max } \approx 0.8$.

The Kolmogorov constant $C$ is also calculated as

$$
C=\max _{\kappa \in\left(0, \kappa_{M}\right)}\left(\frac{E(\kappa) \kappa^{5 / 3}}{\varepsilon^{2 / 3}}\right)
$$

For $\operatorname{Re}(0)=2,000$ and $\bar{t} \simeq 0.63, C \simeq 1.932, Q_{\max } \simeq 0.73$, whereas for $\bar{t} \simeq 0.69, C \simeq 1.92, Q_{\max } \simeq 0.72$. For $\operatorname{Re}(0)=3,000$ at the end of simulation (i.e. $\bar{t} \simeq 0.67$ ), $C \simeq 1.941, Q_{\max } \simeq 0.75$, namely $C$ and $Q_{\max }$ agree with the corresponding quantities known from the literature.

For $\operatorname{Re}(0)=2,000$, Fig. 8a shows $\lambda$ calculated with Eq. (42). For $t=0, \lambda$ follows the variations of $f$, whereas for $t>0$, the mechanism of energy cascade causes sizable increments of $\lambda$ and of its slope especially near the origin. When $t \simeq 0.6, f-1 \approx O\left(r^{2 / 3}\right)$, thus $\lambda \approx r^{-2 / 3}$. Therefore, the Richardson's diffusivity associated to the relative motion of two particles, $D_{R} \propto \lambda r^{2}$ [4], here satisfies the famous Richardson's scaling law $D_{R} \approx r^{4 / 3}[4]$.

In the Figs. $8 \mathrm{~b}$ and $8 \mathrm{c}$, skewness and flatness of $\Delta u_{r}$ are shown for $\bar{t}=0$ and 0.6 , at $\operatorname{Re}(0)=2000 . H_{3}$ is first calculated with Eq. (44), and thereafter $H_{4}$ is determined using Eq. (56). At $\bar{t}=0,\left|H_{3}\right|$ starts from $3 / 7$ 


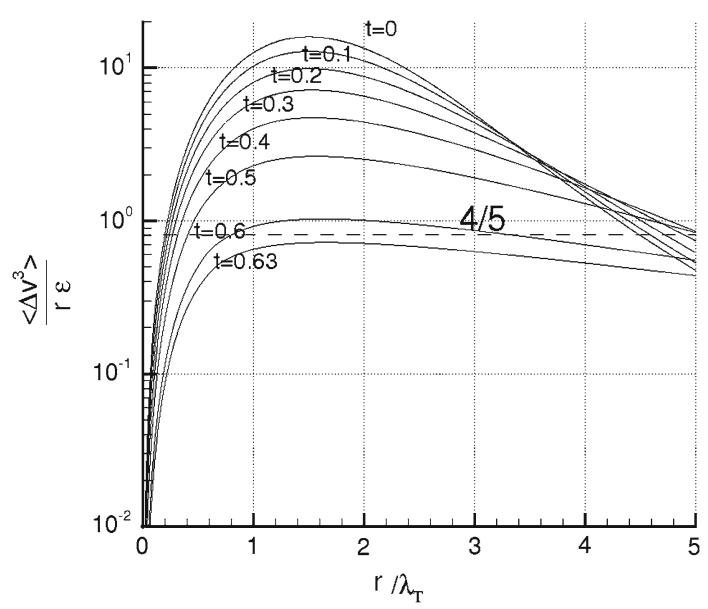

Fig. 7 The Kolmogorov function versus $r / \lambda_{T}$. at $\operatorname{Re}(0)=2,000$, for different times of simulation. The dashed line indicates the value $4 / 5$

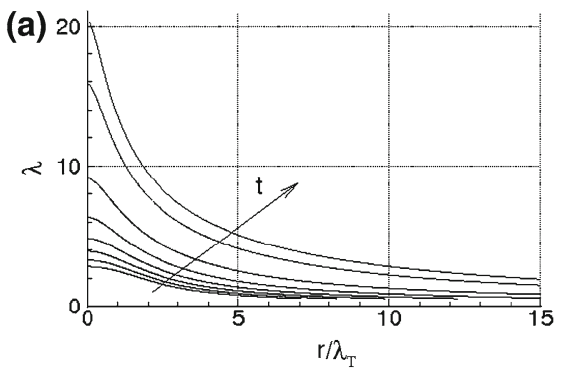

(b)

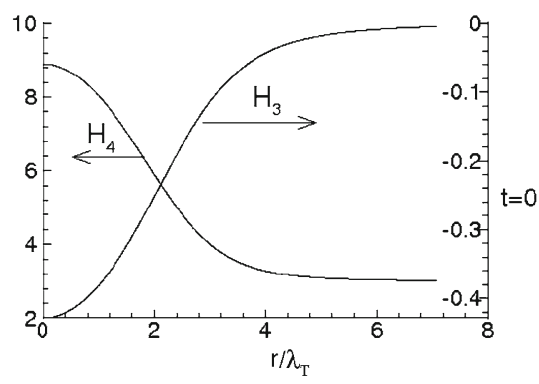

(c)

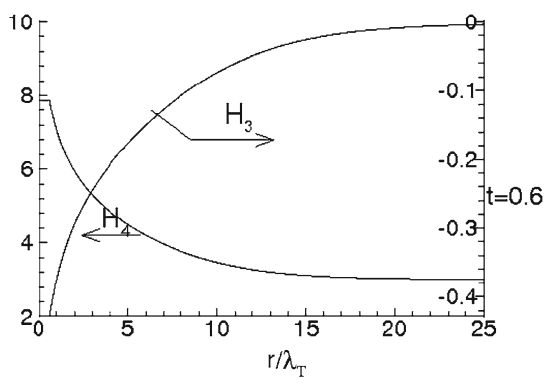

Fig. 8 a Maximum finite size Lyapunov exponent calculated at $\operatorname{Re}(0)=2,000$, for $\bar{t}=0,0.1,0.2,0.3,0.4,0.5,0.6,0.63$; $\mathbf{b}$ and c skewness and Flatness versus $r / \lambda_{T}$ at $\bar{t}=0$ and $\bar{t}=0.6$, respectively

with small slope at $r=0$, then decays until reaching small values. $H_{4}$ also exhibits small derivatives near the origin, where $H_{4} \gg 3$, thereafter it decreases more rapidly than $\left|H_{3}\right|$. Due to the energy cascade, for $\bar{t}=0.6$, the diagram importantly changes, showing different shapes. $\lambda_{T}$ and $R_{\lambda}$ are both changed, so that $H_{3}$ and $H_{4}$ 

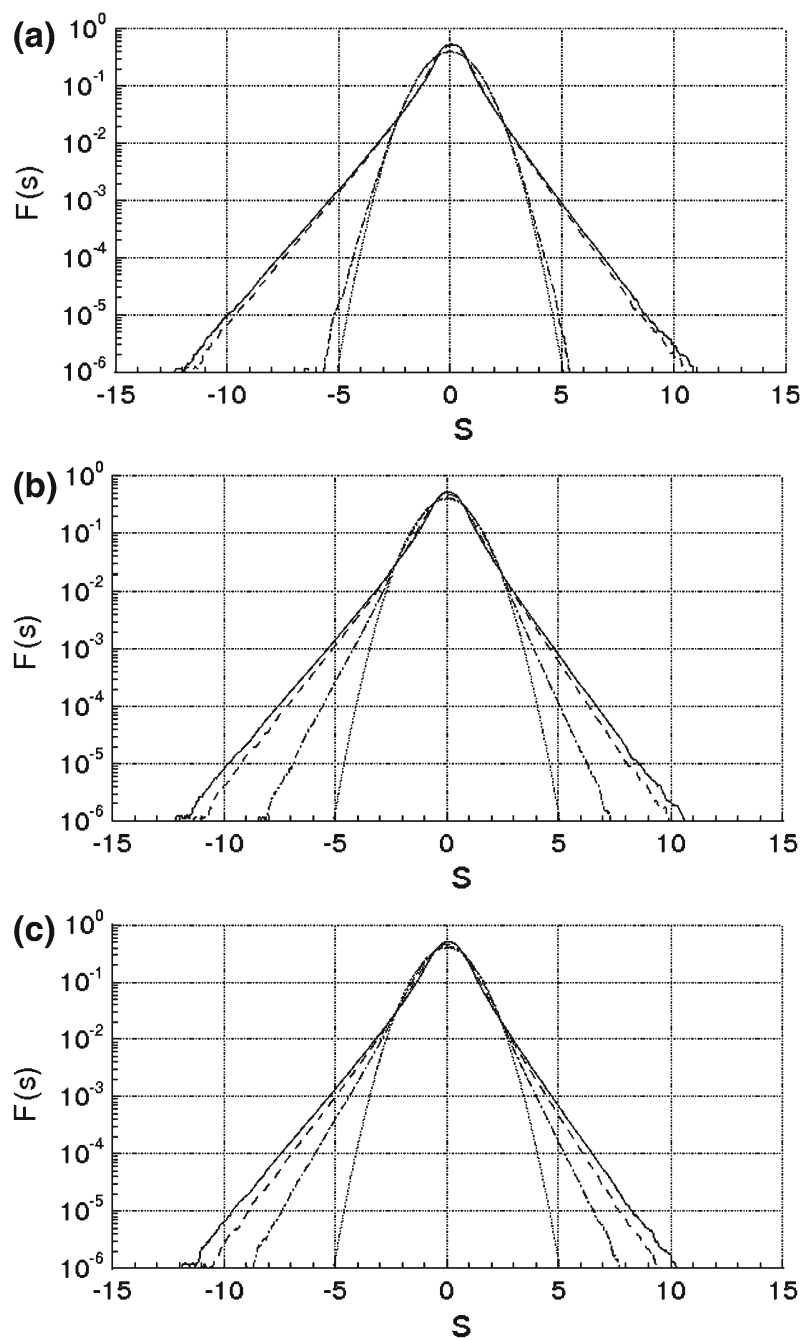

Fig. 9 PDF of the velocity difference fluctuations at the times $\bar{t}=0(\mathbf{a}), \bar{t}=0.5$ (b) and $\bar{t}=0.6(\mathbf{c})$. Continuous lines are for $r=0$, dashed lines are for $r / \lambda_{T}=1$, dot-dashed lines are for $r / \lambda_{T}=5$, dotted lines are for gaussian PDF

vary in smaller distances $r$. Nevertheless, these variations correspond to dimensionless distances $r / \lambda_{T}$ higher than those for $t=0$. Again $\mathrm{H}_{4}-3$ tends to zero more rapidly than $\mathrm{H}_{3}$.

The PDFs of $\Delta u_{r}$, calculated with Eq. (60) for $\operatorname{Re}(0)=2000$, are shown in Fig. 9 in terms of the dimensionless abscissa

$$
s=\frac{\Delta u_{r}}{\left\langle\left(\Delta u_{r}\right)^{2}\right\rangle^{1 / 2}}
$$

in order that their standard deviations are equal to the unity. The figure represents the PDFs for several values of $r / \lambda_{T}$, at $\bar{t}=0,0.5$ and 0.6, where the dotted curves represent the gaussian PDF. $H_{3}(r)$ is first calculated with Eq. (44), then $\psi\left(r, R_{\lambda}\right)$ is identified through Eq. (58), and finally the PDF is obtained with Eq. (60). For $t=0$ (see Fig. 9a) and according to the evolutions of $H_{3}$ and $H_{4}$, the PDFs calculated for $r / \lambda_{T}=0$ and 1 , are quite similar each other, whereas for $r / \lambda_{T}=5$, the PDF is almost a gaussian. Toward the end of simulation, (see Fig. 9b and c), the PDFs for $r / \lambda_{T}=0$ and 1, exhibit more sizable differences, whereas for $r / \lambda_{T}=5$, the PDF differs very much from a gaussian distribution function. This is in line with the plots of $H_{3}(r)$ and $H_{4}(r)$ of Fig. 8.

Next, the spatial structure of $\Delta u_{r}$, given by Eq. (55), is analyzed using the previous results. According to the various works [33,34], $\Delta u_{r}$ behaves quite similarly to a multifractal system, where $\Delta u_{r}$ obeys to a law of the kind $\Delta u_{r}(r) \approx r^{q}$ where the exponent $q$ is a fluctuating function of the space coordinates. This implies that the statistical moments of $\Delta u_{r}(r)$ are expressed through the peculiar scaling exponents $\zeta(P)$. 

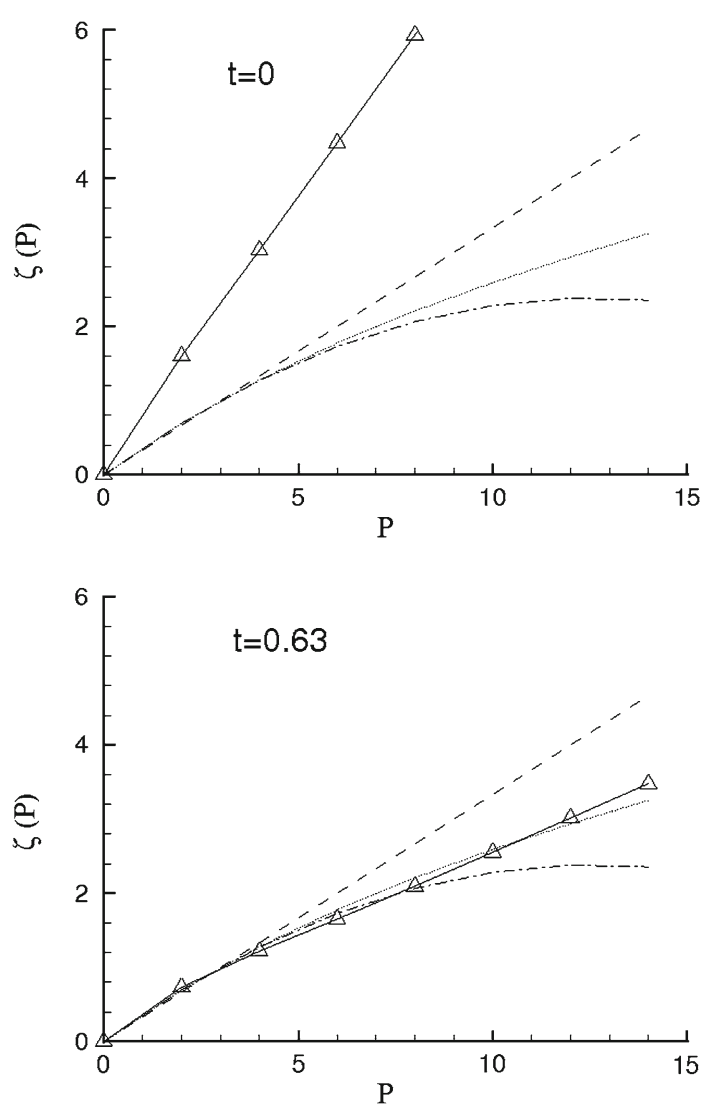

Fig. 10 Scaling exponents of the longitudinal velocity difference versus the order moment at different times, $\operatorname{Re}(0)=2000$. Continuous lines with solid symbols are for the present data. Dashed lines are for Kolmogorov K41 data [5]. Dash dotted lines are for Kolmogorov K62 data [33]. Dotted lines are for She-Leveque data [34]

$$
\left\langle\left(\Delta u_{r}\right)^{P}(r)\right\rangle=A_{P} r^{\zeta(P)}
$$

These scaling exponents are here identified in function of $P$ through a best fitting procedure, in the interval $\left(a, a+\lambda_{T}\right)$, where the endpoints $a$ is an unknown quantity which has to be determined. The location of this interval varies with the time. The calculation of $a, \zeta_{P}$ and $A_{P}$ is carried out through a minimum square method which, for each moment order, is applied to the following optimization problem

$$
J_{P}\left(\zeta_{P}, A_{P}\right) \equiv \int_{a}^{a+\lambda_{T}}\left(\left\langle\left(\Delta u_{r}\right)^{P}\right\rangle-A_{P} r^{\zeta(P)}\right)^{2} d r=\min , \quad P=1,2, \ldots
$$

where $\left(\left\langle\Delta u_{r}^{P}\right)\right\rangle$ are calculated with Eqs. (56), and $a$ is calculated in order to obtain $\zeta(3)=1$.

Figure 10 shows the comparison between the scaling exponents here obtained (continuous lines with solid symbols) and those of the Kolmogorov theories K41 [5] (dashed lines) and K62 [33] (dash dotted lines), and those given by She-Leveque [34] (dotted curves). At $t=0, \zeta(P)$ is the result of the chosen initial condition. For $t>0$, the effect of energy cascade of Eq. (43), causes variations in the statistical moments of $\Delta u_{r}(r)$, thus $\zeta(P)$ gradually diminishes and exhibits a variable slope depending on $P$, until to reach the situation shown in Fig. 10b, where the simulation is just ended. There, $\zeta(P) \simeq P / 3$ for $P<4$, whereas for $P>4 \zeta(P)$ seems to be in agreement with the data of She-Leveque. More in detail, Table 1 reports $\zeta(P)$ for $\bar{t}=0.63$. These values of $\zeta(P)$ and the peculiar diagram of Fig. 10 are the consequence of the spatial variations of $H_{3}(r)$ (see Eq. (44)), caused in turn by the energy cascade, and of the quadratic terms due to the inertia and pressure forces into the expression of $\Delta u_{r}$, which make $\left\langle\left(\Delta u_{r}\right)^{P}\right\rangle$ quite similar to a multifractal system.

Other simulations with different initial correlation functions and Reynolds numbers have been carried out, and all of them lead to analogous results, in the sense that, at the end of the simulations, the diverse quantities 
Table 1 Scaling exponents of the longitudinal velocity difference for different initial Reynolds numbers

\begin{tabular}{|c|c|c|c|c|c|c|c|c|c|c|c|c|c|c|c|}
\hline$\overline{\operatorname{Re}(0)}$ & $\mathrm{P}$ & 1 & 2 & 3 & 4 & 5 & 6 & 7 & 8 & 9 & 10 & 11 & 12 & 13 & 14 \\
\hline 2,000 & $\zeta(\mathrm{P})$ & 0.36 & 0.71 & 1.00 & 1.19 & 1.41 & 1.61 & 1.84 & 2.04 & 2.25 & 2.49 & 2.72 & 2.93 & 3.15 & 3.38 \\
\hline 3,000 & $\zeta(\mathrm{P})$ & 0.36 & 0.72 & 1.00 & 1.19 & 1.43 & 1.64 & 1.84 & 2.03 & 2.25 & 2.49 & 2.71 & 2.92 & 3.11 & 3.33 \\
\hline
\end{tabular}

Table 2 Dimensionless statistical moments of $F\left(\partial u_{r} / \partial r\right)$ at different Taylor scale Reynolds numbers. P.R. as for "present results"

\begin{tabular}{llllr}
\hline $\begin{array}{l}\text { Moment } \\
\text { order }\end{array}$ & $\begin{array}{l}R_{\lambda} \approx 10 \\
\text { P. R. }\end{array}$ & $\begin{array}{l}R_{\lambda}=10^{2} \\
\text { P. R. }\end{array}$ & $\begin{array}{l}R_{\lambda}=10^{3} \\
\text { P. R. }\end{array}$ & $\begin{array}{c}\text { Gaussian } \\
\text { moment }\end{array}$ \\
\hline 3 & -.428571 & -.428571 & -.428571 & 0 \\
4 & 3.96973 & 7.69530 & 8.95525 & 3 \\
5 & -7.21043 & -11.7922 & -12.7656 & 0 \\
6 & 42.4092 & 173.992 & 228.486 & 15 \\
7 & -170.850 & -551.972 & -667.237 & 0 \\
8 & 1035.22 & 7968.33 & 11648.2 & 105 \\
9 & -6329.64 & -41477.9 & -56151.4 & 0 \\
10 & 45632.5 & 617583. & 997938. & 945 \\
\hline
\end{tabular}

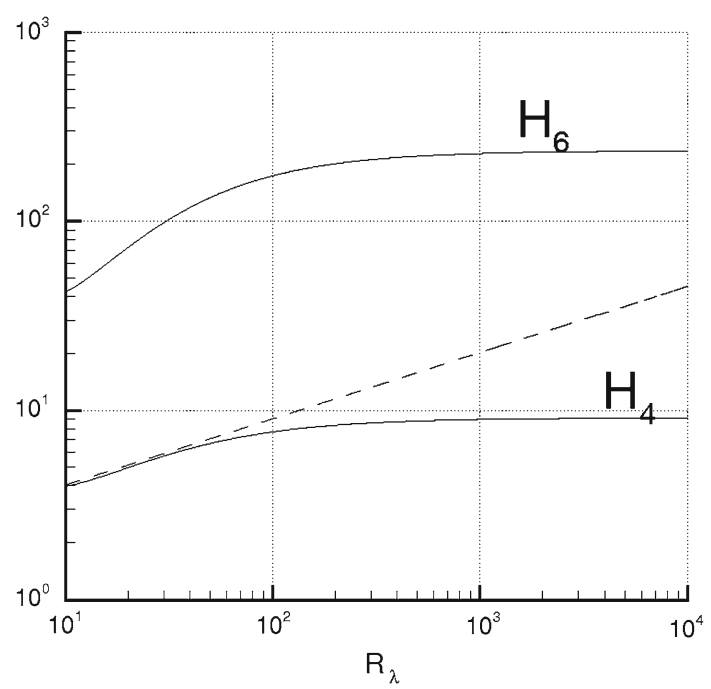

Fig. 11 Dimensionless moments $H_{4}(0)$ and $H_{6}(0)$ plotted vs. $R_{\lambda}$. Continuous lines are for the present results. The dashed line is the tangent to the curve of $H_{4}(0)$ in $R_{\lambda} \approx 10$

such as $Q(r), C$ and $\zeta(P)$ are quite similar to those just calculated. For what concerns the Reynolds number, its increase causes an increment of the Kolmogorov range and smaller dissipation energy rates in accordance to Eq. (76).

In order to study the evolution of the intermittency vs. the Reynolds number, Table 2 gives the first ten statistical moments of $F\left(\partial u_{r} / \partial r\right)$. These are calculated with Eqs. (56) and (57), for $R_{\lambda}=10.12,100$ and 1,000, and are shown in comparison with those of a gaussian distribution function. It is apparent that the constancy of $H_{3}(0)$, causes an intermittency rising with $R_{\lambda}$ (see Eq. (55)). More specifically, Fig. 11 shows $H_{4}(0)$ and $H_{6}(0)$ (continuous lines) in terms of $R_{\lambda}$, calculated with Eqs. (56) and (57), being $H_{3}(0)=-3 / 7$. These moments rise with $R_{\lambda}$ for $10<R_{\lambda}<700$, whereas for $R_{\lambda}>700$ these tend to the saturation and this behavior also happens for the other absolute moments. According to Eq. (56), for $10<R_{\lambda}<70, H_{4}$ and $H_{6}$ are about proportional to $R_{\lambda}^{0.34}$ and $R_{\lambda}^{0.78}$, respectively, and the intermittency increases with the Reynolds number until $R_{\lambda} \approx 700$, where it ceases to rise so quickly. This behavior, represented by the continuous lines, depends on the fact that $\psi \approx \sqrt{R_{\lambda}}$, and results to be in very good agreement with the data of Pullin and Saffman [35], for $10<R_{\lambda}<100$.

Figure 11 can be compared with the data collected by Sreenivasan and Antonia [30], which are here reported into Fig. 12. These latter are referred to several measurements and simulations obtained in different situations which can be very far from the isotropy and homogeneity conditions. Nevertheless the present results and those of Ref. [30] reveals some common elements. According to Ref. [30], $H_{4}$ monotonically rises with $R_{\lambda}$ 


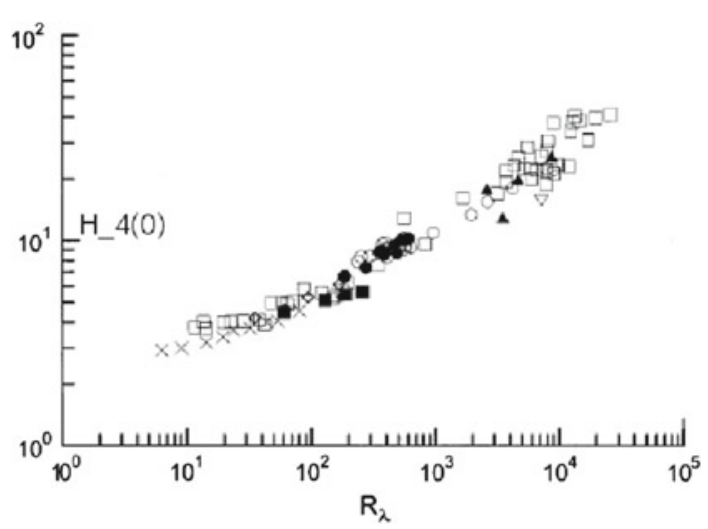

Fig. 12 Flatness $H_{4}(0)$ vs. $R_{\lambda}$. These data are from Ref.[30]

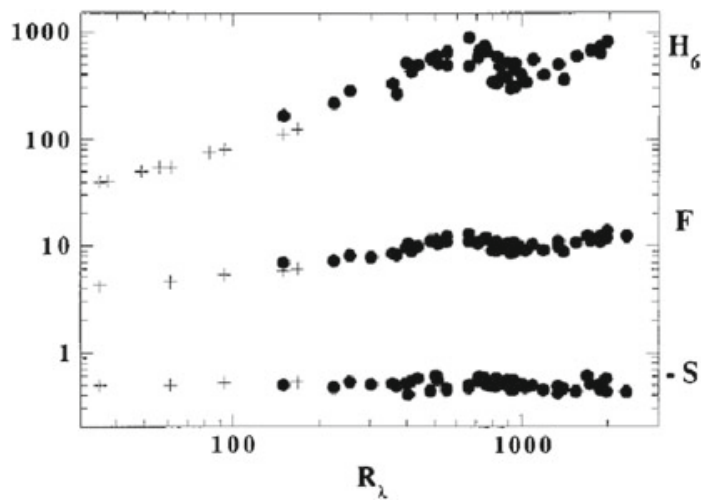

Fig. 13 Skewness $S=H_{3}(0)$, Flatness $F=H_{4}(0)$ and hyperflatness $H_{6}(0)$ vs. $R_{\lambda}$. These data are from Ref. [29]

with a rate which agrees with Eq. (57) for $10<R_{\lambda}<60$ (dashed line, Fig. 11), whereas $H_{3}$ seems to exhibit minor variations. Thereafter, $H_{4}$ continues to rise with about the same rate, without the saturation observed in Fig. 11. The weaker intermittency calculated with the present analysis seems arise from the isotropy which makes $u_{r}$ a gaussian random variable, while, as seen in Sect. 5, without the isotropy, the flatness of $u_{r}$ and $\Delta u_{r}$ can be much greater than that of the isotropic case.

The obtained results are also compared with the data of Tabeling et al. [28,29]. There, in an experiment using low temperature helium gas between two counter-rotating cylinders (closed cell), the authors measure the PDF of $\partial u_{r} / \partial r$ and its moments. Again, the flow could be quite far from the isotropy condition, since these experiments pertain wall-bounded flows, where the walls could importantly influence the fluid velocity in proximity of the probe. The authors found that moments $H_{p}$, with $p>3$, first increase with $R_{\lambda}$ until $R_{\lambda} \approx$ 700 , then exhibit a lightly non-monotonic evolution, and finally cease their variations, denoting a transition behavior (See Fig. 13). As far as the skewness is concerned, the authors observe small percentage variations. Although the isotropy does not describe the non-monotonic evolution near $R_{\lambda}=700$, the results obtained with Eq. (55) can be considered comparable with the data of Refs. [28,29]. The proposed analysis gives again a weaker intermittency with respect to Refs. [28,29].

The normalized PDFs of $\partial u_{r} / \partial r$, calculated with Eq. (60), are shown in Fig. 14 in terms of $s$, here defined as

$$
s=\frac{\partial u_{r} / \partial r}{\left\langle\left(\partial u_{r} / \partial r\right)^{2}\right\rangle^{1 / 2}}
$$

Figure 14a is obtained for $R_{\lambda}=15,30$ and 60, where the PDFs vary, being $H_{3}(0)=-3 / 7$.

As well as in Ref. [29], Figs. 14b and 14c give the PDF for $R_{\lambda}=255,416,514,1035$ and 1553, where $R_{\lambda}$ is calculated, according to Ref. [29], through the Kolmogorov function, with $H_{3}(0)=-3 / 7$. In particular, Fig. 14c represents the enlarged region of Fig. 14b, where the tails of PDF are shown for $5<s<8$. According to Eq. (55), the tails of the PDF rise in the interval $10<R_{\lambda}<700$, whereas for $R_{\lambda}>700$, smaller variations occur. Although the trend observed in Fig. 15 [29] is non-monotonic, Fig. 14c shows that the values of the 

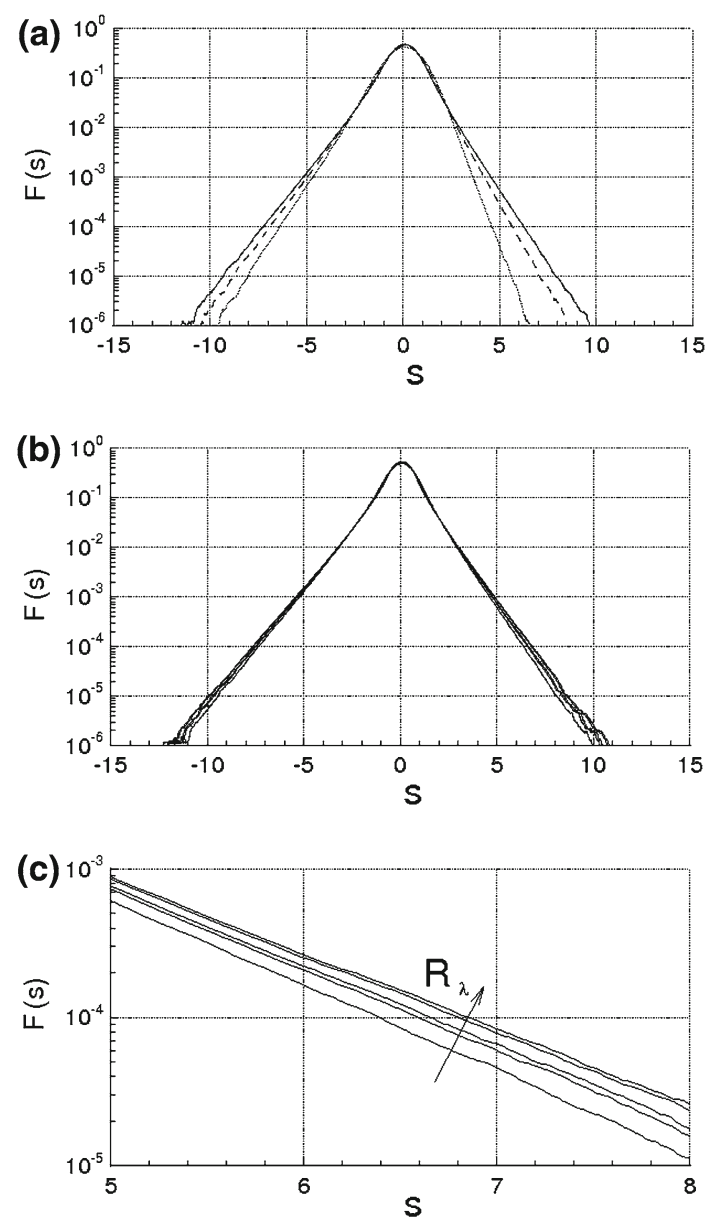

Fig. 14 Log linear plot of the PDF of $\partial u_{r} / \partial r$ for different $R_{\lambda}$. a Dotted, dash dotted and continuous lines are for $R_{\lambda}=15,30$ and 60, respectively. $\mathbf{b}$ and $\mathbf{c}$ PDFs for $R_{\lambda}=255,416,514,1,035$ and 1,553. $\mathbf{c}$ represents an enlarged part of the diagram (b)

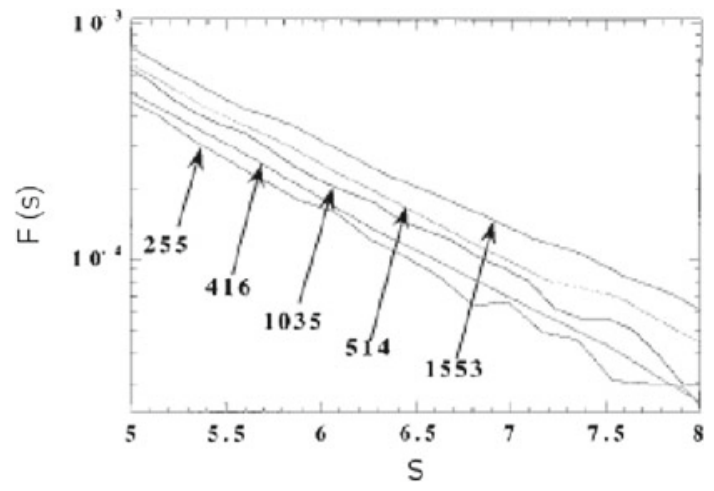

Fig. 15 PDF of $\partial u_{r} / \partial r$ for $R_{\lambda}=255,416,514,1,035$ and 1,553. These data are from Ref. [29]

PDFs calculated with the proposed analysis, for $5<s<8$, exhibit the same order of magnitude of those obtained by Tabeling et al. [29].

Asymmetry and intermittency of the distribution functions are also represented through the integrand function of the 4th order moment of PDF, which is $J_{4}(s)=s^{4} F(s)$. This function is shown in terms of $s$, in Fig. 16, for $R_{\lambda}=15,30$ and 60 . 


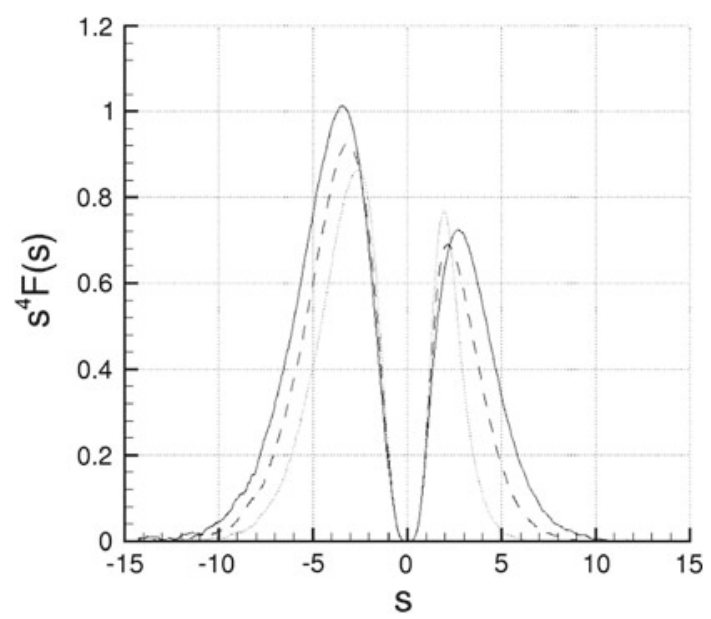

Fig. 16 Plot of the integrand $s^{4} F(s)$ for different $R_{\lambda}$. Dotted, dash dotted and continuous lines are for $R_{\lambda}=15,30$ and 60, respectively

\section{Conclusions}

The proposed analysis, based on the conjecture that the kinematic of the relative motion is much more rapid than the fluid state variables, leads to the closure of the von Kármán-Howarth equation and determines the statistics of $\Delta u_{r}$ by means of the Lyapunov theory. The main limitation of this analysis is that it only studies the developed homogeneous isotropic turbulence and does not consider the intermediate stages of the turbulence.

The results can be here summarized:

1. The momentum equations, written using the referential coordinates, allow to factorize the velocity fluctuation and to express it in Lyapunov exponential form of the local fluid deformation. As a result, the velocity fluctuation is the combined effect of the exponential growth rate and of the rotations of the Lyapunov basis with respect to the fixed frame of reference.

2. The finite scale Lyapunov analysis of the relative motion provides an explanation of the physical mechanism of energy cascade and gives the closure of the von Kármán-Howarth equation. This is a non-diffusive closure expressing $K$ in terms of $f$ and on its spatial gradient.

The fluid incompressibility is a sufficient condition to state that the inertia forces transfer the kinetic energy between the length scales without changing the total kinetic energy. This determines that the skewness $H_{3}(0)$ is constant and that the energy cascade mechanism does not depend on the Reynolds number.

3. The statistics of $\Delta u_{r}$ can be inferred looking at the Fourier series of the velocity difference. This is a nonGaussian statistics, where the constancy of $H_{3}(0)$ implies that $H_{p}(0),(P>3)$ increases with the Taylor scale Reynolds number.

4. The proposed closure of the von Kármán-Howarth equation shows that the mechanism of energy cascade generates energy spectra which satisfy the continuity equation and which follow the Kolmogorov law $\kappa^{-5 / 3}$ in an opportune range of wave-numbers.

5. For developed energy spectrum, the Kolmogorov function exhibits, in an opportune range of $r$, small variations, and its maximum is quite close to $4 / 5$, whereas the Kolmogorov constant is about equal to 1.93 . As the consequence, $\lambda$ and the corresponding diffusivity coefficient vary according to the Richardson law when $r \approx \lambda_{T}$.

6. The analysis also determines the scaling exponents of the moments of the longitudinal velocity difference through a best fitting procedure. For developed energy spectrum, these exponents show variations consistent with those present in the literature. In particular, $\zeta(P) \simeq P / 3$ for $P<4$, whereas for higher $P, \zeta(P)$ exhibits multiscaling behavior.

Acknowledgments This work was partially supported by the Italian Ministry for the Universities and Scientific and Technological Research (MIUR).

Open Access This article is distributed under the terms of the Creative Commons Attribution Noncommercial License which permits any noncommercial use, distribution, and reproduction in any medium, provided the original author(s) and source are credited. 


\section{Appendix A}

The von Kármán-Howarth equation gives the evolution in the time of the longitudinal correlation function for isotropic turbulence. The correlation function of the velocity components is the symmetrical second order tensor $R_{i j}(\mathbf{r})=\left\langle u_{i} u_{j}^{\prime}\right\rangle$, where $u_{i}$ and $u_{j}^{\prime}$ are the velocity components at $\mathbf{x}$ and $\mathbf{x}+\mathbf{r}$, respectively, being $\mathbf{r}$ the separation vector. The equations for $R_{i j}$ are obtained by the Navier-Stokes equations written in the two points $\mathbf{x}$ and $\mathbf{x}+\mathbf{r}$ [6,7]. For isotropic turbulence $R_{i j}$ can be expressed as

$$
R_{i j}(\mathbf{r})=u^{2}\left[(f-g) \frac{r_{i} r_{j}}{r^{2}}+g \delta_{i j}\right]
$$

$f$ and $g$ are, respectively, longitudinal and lateral correlation functions, which are

$$
f(r)=\frac{\left\langle u_{r}(\mathbf{x}) u_{r}(\mathbf{x}+\mathbf{r})\right\rangle}{u^{2}}, \quad g(r)=\frac{\left\langle u_{n}(\mathbf{r}) u_{n}(\mathbf{x}+\mathbf{r})\right\rangle}{u^{2}}
$$

where $u_{r}$ and $u_{n}$ are, respectively, the velocity components parallel and normal to $\mathbf{r}$, whereas $r=|\mathbf{r}|$ and $u^{2}=\left\langle u_{r}^{2}\right\rangle=\left\langle u_{n}^{2}\right\rangle=1 / 3\left\langle u_{i} u_{i}\right\rangle$. Due to the continuity equation, $f$ and $g$ are linked each other by the relationship

$$
g=f+\frac{1}{2} \frac{\partial f}{\partial r} r
$$

The von Kármán-Howarth equation reads as follows [6,7]

$$
\frac{\partial f}{\partial t}=\frac{K}{u^{2}}+2 v\left(\frac{\partial^{2} f}{\partial r^{2}}+\frac{4}{r} \frac{\partial f}{\partial r}\right)-10 v \frac{\partial^{2} f}{\partial r^{2}}(0) f
$$

where $K$ is an even function of $r$, defined as [6,7]

$$
\left(r \frac{\partial}{\partial r}+3\right) K(r)=\frac{\partial}{\partial r_{k}}\left\langle u_{i} u_{i}^{\prime}\left(u_{k}-u_{k}^{\prime}\right)\right\rangle
$$

and which can also be expressed in terms of the longitudinal triple correlation function $k(r)=\left\langle u_{r}^{2}(\mathbf{x}) u_{r}\right.$ $(\mathbf{x}+\mathbf{r})\rangle / u^{3}$

$$
K(r)=u^{3}\left(\frac{\partial}{\partial r}+\frac{4}{r}\right) k(r)
$$

The boundary conditions of Eq. (72) are [6,7]

$$
f(0)=1, \lim _{r \rightarrow \infty} f(r)=0
$$

The viscosity is responsible for the decay of the turbulent kinetic energy, whose rate is $[6,7]$

$$
\frac{d u^{2}}{d t}=10 v u^{2} \frac{\partial^{2} f}{\partial r^{2}}(0)
$$

This energy is distributed at different wave-lengths according to the energy spectrum $E(\kappa)$ which is calculated as the Fourier Transform of $f u^{2}$, whereas the "transfer function" $T(\kappa)$ is the Fourier Transform of $K$ [7], i.e.

$$
\left[\begin{array}{c}
E(\kappa) \\
T(\kappa)
\end{array}\right]=\frac{1}{\pi} \int_{0}^{\infty}\left[\begin{array}{c}
u^{2} f(r) \\
K(r)
\end{array}\right] \kappa^{2} r^{2}\left(\frac{\sin \kappa r}{\kappa r}-\cos \kappa r\right) d r
$$

where $\kappa=|\kappa|$ and $T(\kappa)$ identically satisfies to the integral condition

$$
\int_{0}^{\infty} T(\kappa) d \kappa=0
$$


which states that $K$ does not modify the total kinetic energy. The rate of energy dissipation $\varepsilon$ is calculated for isotropic turbulence as follows [7]

$$
\varepsilon=-\frac{3}{2} \frac{d u^{2}}{d t}=2 v \int_{0}^{\infty} \kappa^{2} E(\kappa) d \kappa
$$

The microscales of Taylor $\lambda_{T}$, and of Kolmogorov $\ell$, are defined as

$$
\lambda_{T}^{2}=\frac{u^{2}}{\left\langle\left(\partial u_{r} / \partial r\right)^{2}\right\rangle}=-\frac{1}{\partial^{2} f / \partial r^{2}(0)}, \quad \ell=\left(\frac{\nu^{3}}{\varepsilon}\right)^{1 / 4}
$$

\section{Appendix B: Critical Reynolds number}

The purpose of this appendix is to provide a rough estimation of the critical Reynolds number assuming that the turbulence, in any case supposed fully developed, homogeneous and isotropic, is caused by the bifurcations of the Navier-Stokes equations [36]. We assume that the bifurcations cascade are expressed in terms of the characteristic scales by the asymptotic approximation [22]

$$
l_{n}=\frac{l_{1}}{\alpha^{n-1}}
$$

where $\alpha \approx 2$ [23,37], and $l_{n}$ represent the average distance between two branches of fixed points which born in the same bifurcation. Equation (81) is supposed to describe the route toward the chaos and is assumed to be valid until the onset of the turbulence. In this situation the minimum for $l_{n}$ cannot be less than the dissipation length or Kolmogorov scale $\ell=\left(v^{3} / \varepsilon\right)^{1 / 4}$ [36], where $l_{1}$ gives a good estimation of the correlation length of the phenomenon [38] which, in this case is the Taylor scale $\lambda_{T}$. Thus, $\ell<l_{n}<\lambda_{T}$, and

$$
\ell=\frac{\lambda_{T}}{\alpha^{N-1}}
$$

where $N$ is the number of bifurcations at the beginning of the turbulence. Equation (82) gives the connection between the critical Reynolds number and number of bifurcations. In fact, the characteristic Reynolds numbers associated to the scales $\ell$ and $\lambda_{T}$ are $R_{K}=\ell u_{K} / \nu \equiv 1$ and $R_{\lambda}=\lambda_{T} u / v$, respectively, where $u_{K}=(\nu \varepsilon)^{1 / 4}$ is characteristic velocity at the Kolmogorov scale, and $u=\sqrt{\left\langle u_{i} u_{i}\right\rangle / 3}$ is the velocity standard deviation [7]. For isotropic turbulence, these scales are linked each other by [7]

$$
\lambda_{T} / \ell=15^{1 / 4} \sqrt{R_{\lambda}}
$$

In view of Eq. (82), this ratio can be also expressed through $N$, i.e.

$$
\alpha^{N-1}=15^{1 / 4} \sqrt{R_{\lambda}}
$$

Assuming that $\alpha$ is equal to the Feigenbaum constant $(2.502 \ldots)$, the value $R_{\lambda} \simeq 1.6$ obtained for $N=2$ is not compatible with $\lambda_{T}$ which is the correlation scale, while the result $R_{\lambda} \simeq 10.12$, calculated for $N=3$, is an acceptable minimum value for $R_{\lambda}$. The order of magnitude of these values can be considered in agreement with the various scenarios describing the roads to the turbulence $[39,37,40,41]$, and with the diverse experiments [42-44] which state that the turbulence begins for $N \geq 3$. Of course, this minimum value for $R_{\lambda}$ is the result of the assumptions $\alpha \simeq 2.502, l_{1} \simeq \lambda_{T}, l_{N} \simeq \ell$ and of the asymptotic approximation (81). 


\section{References}

1. Ottino, J.M.: The Kinematics of Mixing: Stretching, Chaos, and Transport. Cambridge Texts in Applied Mathematics, New York (1989)

2. Ottino, J.M.: Mixing, chaotic advection, and turbulence. Annu. Rev. Fluid Mech. 22, 207-253 (1990)

3. Truesdell, C.: A First Course in Rational Continuum Mechanics. Academic, New York (1977)

4. Richardson, L.F.: Atmospheric diffusion shown on a distance-neighbour graph. Proc. Roy. Soc. Lond A 110, 709 (1926)

5. Kolmogorov, A.N.: Dissipation of energy in locally isotropic turbulence. Dokl. Akad. Nauk SSSR 32(1), 19-21 (1941)

6. von Kármán, T., Howarth, L.: On the statistical theory of isotropic turbulence. Proc. Roy. Soc. A 164(14), 192 (1938)

7. Batchelor, G.K.: The Theory of Homogeneous Turbulence. Cambridge University Press, Cambridge (1953)

8. Eyink, G.L., Sreenivasan, K.R.: Onsager and the theory of hydrodynamic turbulence. Rev. Mod. Phys. 78, 87-135 (2006)

9. Hasselmann, K.: Zur Deutung der dreifachen Geschwindigkeitskorrelationen der isotropen Turbulenz. Dtsch. Hydrogr. Z 11(5), 207-217 (1958)

10. Millionshtchikov, M.: Isotropic turbulence in the field of turbulent viscosity. JETP Lett. 8, 406-411 (1969)

11. Oberlack, M., Peters, N.: Closure of the two-point correlation equation as a basis for Reynolds stress models. Appl. Sci. Res. 51, 533-539 (1993)

12. Skorokhod, A.V.: Stochastic Equations for Complex Systems. Springer, Berlin (1988)

13. Risken, H.: The Fokker-Planck Equation: Methods of Solution and Applications. Springer, Berlin (1989)

14. Domaradzki, J.A., Mellor, G.L.: A simple turbulence closure hypothesis for the triple-velocity correlation functions in homogeneous isotropic turbulence. Jour. Fluid Mech. 140, 45-61 (1984)

15. Onufriev, A.: On a model equation for probability density in semi-empirical turbulence transfer theory. In: The Notes on Turbulence. Nauka, Moscow (1994)

16. Grebenev, V.N., Oberlack, M.: A Chorin-type formula for solutions to a closure model for the von Kármán-Howarth Equation. J. Nonlinear Math. Phys. 12(1), 19 (2005)

17. Grebenev, V.N., Oberlack, M.: A Geometric interpretation of the second-Order structure function arising in turbulence. Math. Phy. Anal. Geom. 12(1), 1-18 (2009)

18. Toth, Z., Kalnay, E.: Ensemble forecasting at NMC: the generation of perturbations. Bull. Amer. Meteor. Soc. 74, 2317-2330 (1993)

19. Kalnay, E., Corazza, M., Cai, M.: Are bred vectors the same as Lyapunov vectors? http://www.atmos.umd.edu/ekalnay/ lyapbredamsfinal.htm (2004)

20. Lu, J., Yang, G., Oh, H., Luo, A.C.J.: Computing Lyapunov exponents of continuous dynamical systems: method of Lyapunov vectors. Chaos Solitons Fractals 23(5), 1879-1892 (2005)

21. Lamb, H.: Hydrodynamics. Dover Publications, USA (1945)

22. Guckenheimer, J., Holmes, P.: Nonlinear Oscillations, Dynamical Systems, and Bifurcations of Vector Fields. Springer, Berlin (1990)

23. Sprott, J.C.: Chaos and Time-series Analysis. Oxford University Press, Oxford (2003)

24. Annan, J.D.: On the Orthogonality of Bred Vectors. Mon. Weather Review. 132(3), 843-849 (2004)

25. Lehmann, E.L.: Elements of Large-Sample Theory. Springer, Berlin (1999)

26. Borisenko, A.I., Tarapov, I.E.: Vector and Tensor Analysis with Applications. Dover Publication, USA (1990)

27. Robertson, H.P.: The invariant theory of isotropic turbulence. Math. Proc. Camb. Ph. Soc. 36, 209-223 (1940)

28. Tabeling, P., Zocchi, G., Belin, F., Maurer, J., Willaime, H.: Probability density functions, skewness, and flatness in large Reynolds number turbulence. Phys. Rev. E 53, 1613 (1996)

29. Belin, F., Maurer, J., Willaime, H., Tabeling, P.: Velocity gradient distributions in fully developed turbulence: an experimental study. Phys. Fluids 9(12), 3843-3850 (1997)

30. Sreenivasan, K.R., Antonia, R.A.: The phenomenology of small-scale turbulence. Annu. Rev. Fluid Mech. 29, 435-472 (1997)

31. Madow, W.G.: Limiting distributions of quadratic and bilinear forms. Ann. Appl. Probab. 11(2), 125-146 (1940)

32. Hildebrand, F.B.: Introduction to Numerical Analysis. Dover Publications, USA (1987)

33. Kolmogorov, A.N.: Refinement of previous hypothesis concerning the local structure of turbulence in a viscous incompressible fluid at high Reynolds number. J. Fluid Mech. 12, 82-85 (1962)

34. She, Z.S., Leveque, E.: Universal scaling laws in fully developed turbulence. Phys. Rev. Lett. 72, 336 (1994)

35. Pullin, D., Saffman, P.: On the Lundgren Townsend model of turbulent fine structure. Phys. Fluids A 5(1), 126 (1993)

36. Landau, L.D., Lifshitz, M.: Fluid Mechanics. Pergamon London, England (1959)

37. Feigenbaum, M.J.: . J. Stat. Phys. 19, (1978)

38. Prigogine, I.: Time, Chaos and the Laws of Chaos. Progress, Moscow (1994)

39. Ruelle, D., Takens, F.: Commun. Math. Phys. 20, 167 (1971)

40. Pomeau, Y., Manneville, P.: Commun. Math. Phys. 74, 189 (1980)

41. Eckmann, J.P.: Roads to turbulence in dissipative dynamical systems. Rev. Mod. Phys. 53, 643-654 (1981)

42. Gollub, J.P., Swinney, H.L.: Onset of turbulence in a rotating fluid. Phys. Rev. Lett. 35, 927 (1975)

43. Giglio, M., Musazzi, S., Perini, U.: Transition to chaotic behavior via a reproducible sequence of period doubling bifurcations. Phys. Rev. Lett. 47, 243 (1981)

44. Maurer, J., Libchaber, A.: Rayleigh-Bénard Experiment in liquid helium: frequency Locking and the onset of turbulence. J. de Physique Lett. 40, L419-L423 (1979) 